\title{
A Virgo Environmental Survey Tracing lonised Gas Emission (VESTIGE)
}

\section{Modeling ram pressure stripping of diffuse gas in the Virgo cluster spiral galaxy NGC 4330*}

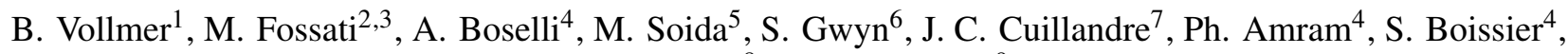 \\ M. Boquien ${ }^{8}$, and G. Hensler ${ }^{9}$ \\ 1 Université de Strasbourg, CNRS, Observatoire astronomique de Strasbourg, UMR 7550, 67000 Strasbourg, France \\ e-mail: Bernd.Vollmer@astro.unistra.fr \\ 2 Dipartimento di Fisica G. Occhialini, Università degli Studi di Milano Bicocca, Piazza della Scienza 3, 20126 Milano, Italy \\ 3 Institute for Computational Cosmology and Center for Extragalactic Astronomy, Durham University, South Road, Durham DH1 \\ 3LE, UK \\ 4 Aix Marseille Université, CNRS, CNES, LAM, Marseille, France \\ 5 Astronomical Observatory, Jagiellonian University, Kraków, Poland \\ ${ }^{6}$ NRC Herzberg Astronomy and Astrophysics, 5071 West Saanich Road, Victoria, BC V9E 2E7, Canada \\ 7 AIM, CEA, CNRS, Université Paris-Saclay, Université Paris Diderot, Sorbonne Paris Cité, Observatoire de Paris, PSL University, \\ 91191 Gif-sur-Yvette Cedex, France \\ 8 Centro de Astronomía (CITEVA), Universidad de Antofagasta, Avenida Angamos 601, Antofagasta 1270300, Chile \\ 9 Department for Astrophysics, University of Vienna, Türkenschanzstrasse 17, 1180 Vienna, Austria
}

Received 27 May 2020 / Accepted 3 November 2020

\section{ABSTRACT}

\begin{abstract}
NGC 4330 is one of the Virgo galaxies whose UV emission distributions show a tail structure. An associated tail structure is also observed in the $\mathrm{HI}$ and $\mathrm{H} \alpha$ emission distributions. Previous dynamical modeling has shown that the galaxy is approaching the cluster center and is therefore undergoing increasing ram pressure stripping. Recent stellar population fitting of deep optical spectra together with multiband photometry led to the determination of the time when star formation was quenched in the galactic disk. We introduce a new version of the dynamical model that includes not only the dense neutral gas, but also the diffuse ionized gas, and we aim to reproduce the $\mathrm{HI}, \mathrm{H} \alpha$, and UV distributions together with the star formation histories of the outer gas-free parts of the galactic disk. We present the results of 50 simulations with five different Lorentzian temporal ram pressure profiles and five different delays between the simulation onset and peak ram pressure. The delays were introduced to study the influence of galactic structure on the outcome of the simulations. The inclusion of diffuse gas stripping significantly changes the HI, UV, and $\mathrm{H} \alpha$ emission distributions. The simulations with diffuse gas stripping naturally led to vertical low surface density filaments in the downwind region of the galactic disk. These filaments occur less frequently in the simulations without diffuse gas stripping. The simulations with diffuse gas stripping led to better joint fits to the spectral energy distributions (SEDs) and optical spectra. The HI, near-UV, and H $\alpha$ morphologies of the model snapshots that best reproduce the SEDs and optical spectra are sufficiently different to permit a selection of best-fit models. We conclude that the inclusion of diffuse gas stripping significantly improves the resemb+lance between the model and observations. Our preferred model yields a time to peak ram pressure of $140 \mathrm{Myr}$ in the future. The spatial coincidence of the radio continuum and diffuse $\mathrm{H} \alpha$ tails suggests that both gas phases are stripped together. We suggest that the star formation in the outer tail is sporadic and low level, and this explains the absence of a significant amount of cosmic ray electrons there. Furthermore, we suggest that the mixed interstellar medium is ionized by collisions with the thermal electrons of the ambient intracluster medium, which confines the filaments.
\end{abstract}

Key words. galaxies: evolution - galaxies: individual: NGC 4330 - galaxies: interactions - galaxies: kinematics and dynamics galaxies: ISM

\section{Introduction}

Once a galaxy has entered a galaxy cluster, its evolution will change, sometimes drastically. Multiple gravitational interactions together with the influence of the cluster potential can remove the stellar and gaseous content of its outer disk (galaxy

^ Based on observations obtained with MegaPrime/MegaCam, a joint project of CFHT and CEA/DAPNIA, at the Canada-French-Hawaii Telescope (CFHT), which is operated by the National Research Council (NRC) of Canada, the Institut National des Sciences de l'Univers of the Centre National de la Recherche Scientifique (CNRS) of France, and the University of Hawaii. harassment; Moore et al. 1996). The rapid motion of the galaxy within the cluster atmosphere, the hot (temperature $T \sim 10^{7} \mathrm{~K}$ ) and tenuous (density $n \sim 10^{-4} \mathrm{~cm}^{-3}$ ) intracluster medium (ICM), causes the removal of the outer gas disk via ram pressure. In contrast to galaxy harassment, ram pressure stripping does not affect the stellar content of the galaxy. Spiral galaxies that underwent or are undergoing ram pressure stripping show a truncated gas disk together with a symmetric stellar disk (e.g., VLA Imaging of Virgo in Atomic gas, VIVA, Chung et al. 2009). If the interaction is ongoing, a gas tail mainly detected in $\mathrm{HI}$ is present (e.g., Chung et al. 2007). The gas truncation radius is set by the galaxy's closest passage to the cluster center via the criterion introduced by Gunn \& Gott (1972): 
$\rho_{\mathrm{ICM}} v_{\mathrm{gal}}^{2}=\pi \Sigma v_{\mathrm{rot}}^{2} / R$

(1)

where $\rho_{\text {ICM }}$ is the ICM density, $v_{\text {gal }}$ the galaxy velocity, $\Sigma$ the surface density of the interstellar medium (ISM), $v_{\text {rot }}$ the rotation velocity of the galaxy, and $R$ the stripping radius. If peak ram pressure occurred more than 400-500 Myr ago, the gas tails have disappeared and the truncated gas disk has become symmetric again (Vollmer et al. 2009).

Under extreme conditions, ram-pressure stripped galaxies often show extraplanar, one-sided optical and UV emission as well as important tails of ionized gas (e.g., Yagi et al. 2010, 2017; Poggianti et al. 2017). Because of their optical appearance, these objects are called jellyfish galaxies. Most of the $\mathrm{H} \alpha$ emission in these tails is due to photoionization by massive stars born in situ in the tails (Poggianti et al. 2019). Extraplanar molecular gas is often found in jellyfish galaxies (Jáchym et al. 2014, 2019; Moretti et al. 2018), from which the ionizing stars are formed.

Once the gas has been removed from the outer galactic disk under the action of ram pressure, the corresponding stellar content remains gas- and dust-free and gradually stops star formation. Optical spectroscopy together with multiwavelength photometry can be used to determine the stellar populations and thus the star formation history (SFH) of the ram-pressure stripped parts of the galactic disk (Boselli et al. 2006; Crowl \& Kenney 2008). These SFHs can then be compared to those of dynamical models. For the Virgo cluster galaxy NGC 4388, an observed quenching timescale of $200 \mathrm{Myr}$ was determined by Pappalardo et al. (2010) based on a Very Large Telescope (VLT) spectrum and optical and UV photometry. An updated dynamical model including ram pressure stripping was able to reproduce the observed quenching timescale together with additional observational characteristics (Vollmer et al. 2018): HI distribution and velocity field as well as far-UV (FUV), $\mathrm{H} \alpha$, and polarized radio continuum emission distribution. A realistic model has to fulfill all these observational constraints. From the model, the ram pressure temporal profile, time to peak ram pressure, and the angle between the galaxy velocity within the cluster and its disk plane can be determined. A model-based time series of ram-pressure stripped galaxies was established by Vollmer et al. (2009). The Virgo spiral galaxy NGC 4330 is part of this time series.

The ISM of a spiral galaxy consists of different phases: (i) cool dense molecular gas $\left(T \sim 10-30 \mathrm{~K}, n \gtrsim 100 \mathrm{~cm}^{-1}\right)$, (ii) cold neutral hydrogen $\left(T \sim 100 \mathrm{~K}, n \sim 100 \mathrm{~cm}^{-1}\right)$, (iii) warm neutral hydrogen $\left(T \sim 8000 \mathrm{~K}, n \sim 1 \mathrm{~cm}^{-1}\right)$, and (iv) warm diffuse ionized hydrogen $\left(T \sim 8000 \mathrm{~K}, n \sim 0.4 \mathrm{~cm}^{-1}\right)$. Since the acceleration of a gas cloud by ram pressure is inversely proportional to the gas surface density (Eq. (1)), the different phases are expected to be stripped with different efficiencies. Indeed, the stripped diffuse ionized gas is accelerated to higher velocities and is therefore observed at larger distances from the host galaxy than the dense gas (Vollmer et al. 2009; Moretti et al. 2018). On the other hand, the stripped dense CO-emitting gas seems to be well mixed with the warm HI (Vollmer et al. 2008, 2012a,b; Jáchym et al. 2014; Nehlig et al. 2016), which is consistent with a scenario where the neutral turbulent ISM is stripped as an entity. We therefore expect that the distribution of the stripped diffuse ionized gas is more extended than that of the neutral gas. The detection of this gas in deep $\mathrm{H} \alpha$ observations represents an important additional constraint on simulations of ram-pressure stripped galaxies. An additional constraint on the simulations comes from the SFH of stripped gas-free regions of the stellar disk. Detailed simulations of a ram-pressure stripped galaxy can thus not only give access to the interaction parameters (see Vollmer et al. 2009), but can also provide insight into the reaction of the different gas phases to ram pressure.

In this article, we (i) test a new version of the dynamical model that includes not only the dense neutral gas, but also the diffuse ionized gas and apply it to NGC 4330 and (ii) aim to reproduce the observed HI, $\mathrm{H} \alpha$, and near-UV (NUV) distributions of NGC 4330 together with the SFHs of the outer gas-free parts of the galactic disk. This article represents a continuation and update of the work of Vollmer et al. (2012a,b).

\section{NGC 4330}

NGC 4330 is one of the Virgo spiral galaxies with a long HI tail that was observed by Chung et al. (2007). It has a maximum rotation velocity of $v_{\text {rot }} \sim 180 \mathrm{~km} \mathrm{~s}^{-1}$ and a total HI mass of $M_{\mathrm{HI}}=4.5 \times 10^{8} M_{\odot}$ (Chung et al. 2009). NGC 4330 is located at a projected distance of $\sim 2^{\circ}(600 \mathrm{kpc})$ from the cluster center (i.e., it is relatively close to $M$ 87) and has a radial velocity of $400 \mathrm{~km} \mathrm{~s}^{-1}$ with respect to the Virgo cluster mean. The HI deficiency of NGC 4330 - which is defined as the logarithm of the ratio of the HI content of a field galaxy of the same morphological type and diameter as the observed HI mass - is 0.8 (Chung et al. 2007), that is to say, the galaxy has lost about $80 \%$ of its atomic hydrogen. The HI distribution in the galactic disk (upper-left panel of Fig. 1) is truncated at about half the optical radius. In addition, NGC 4330 is one of the rare Virgo galaxies that show extended UV tails (Abramson et al. 2011). The HI and UV tails show a significant offset, with the HI tail being downwind of the UV tail. At the leading edge of the interaction, the $\mathrm{H} \alpha$ emission and dust extinction distribution bend sharply out of the galactic disk. These features are signs of active ram pressure stripping. Roediger \& Hensler (2005) elaborated on the subsequent stripping phases for face-on ram pressure stripping. NGC 4330 undergoes the instantaneous stripping phase where the outer part of the gas disk is displaced but only partially unbound. This leads to a straightly bent but coherent outer gas disk.

Vollmer et al. (2012a,b) presented a detailed dynamical model for NGC 4330. Their best-fit model qualitatively reproduced the observed projected position, the radial velocity of the galaxy, the molecular and atomic gas distribution and velocity field, and the UV distribution in the region where a gas tail is present. In contrast to other Virgo spiral galaxies affected by ram pressure stripping, NGC 4330 does not show an asymmetric ridge of polarized radio continuum emission. This is due to the particular projection of NGC 4330, where the compressed large-scale magnetic fields are oriented along the light-of-sight. Vollmer et al. (2012a,b) concluded that the ISM is stripped as a whole entity and that only a tiny fraction of dense clouds can be left behind. Extraplanar star formation proceeds in dense gas arms pushed by ram pressure. The collapsing clouds decouple rapidly from the ram pressure wind, and the young, UV-emitting stars stay behind the gas.

On the basis of dynamical models, the galaxy moves toward the north and continues to approach the cluster center, with the closest approach occurring in $\sim 100$ Myr. Despite the success of their model, Vollmer et al. (2012a,b) noted that the observed red UV color on the windward side was not reproduced by the model. Moreover, their simulations could only produce the model distribution of HII regions, that is, compact regions of high ionized gas density and high $\mathrm{H} \alpha$ surface brightness (Fig. 9 of Vollmer et al. 2012a,b). Since the diffuse ionized gas is much less dense than the gas in the HII regions, it has a much lower 


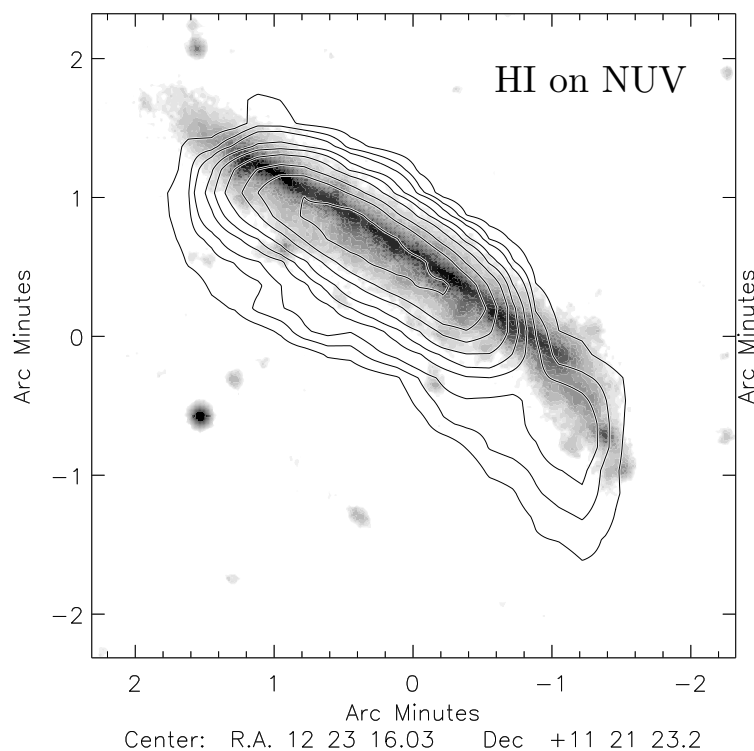

(a)

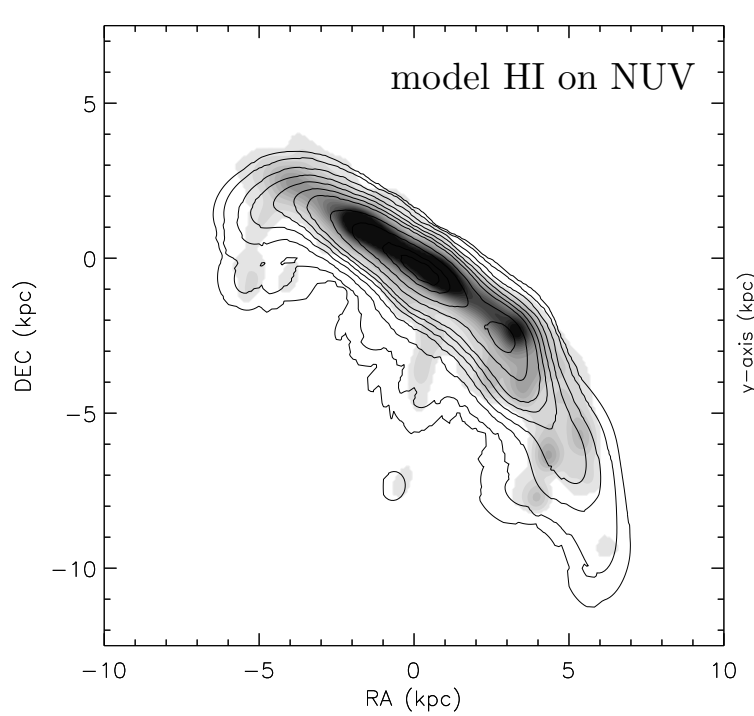

(c)

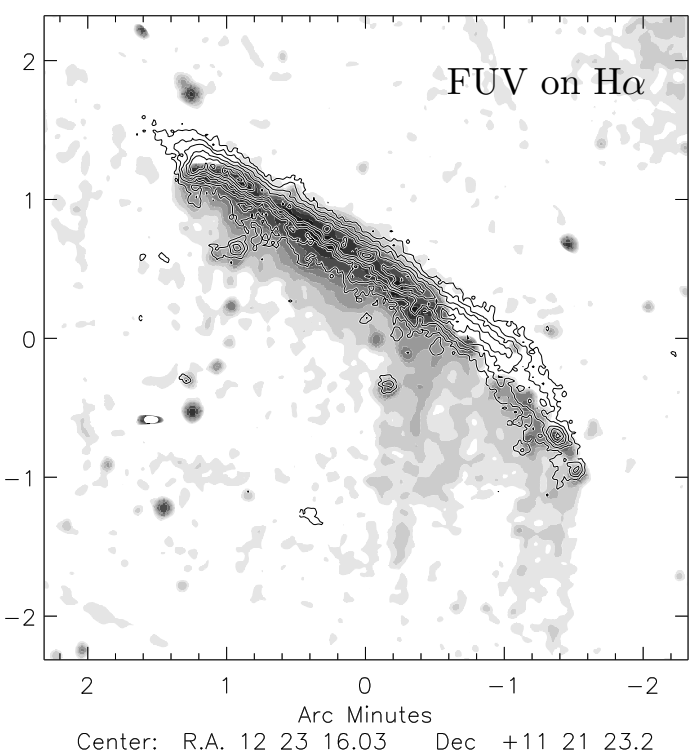

(b)

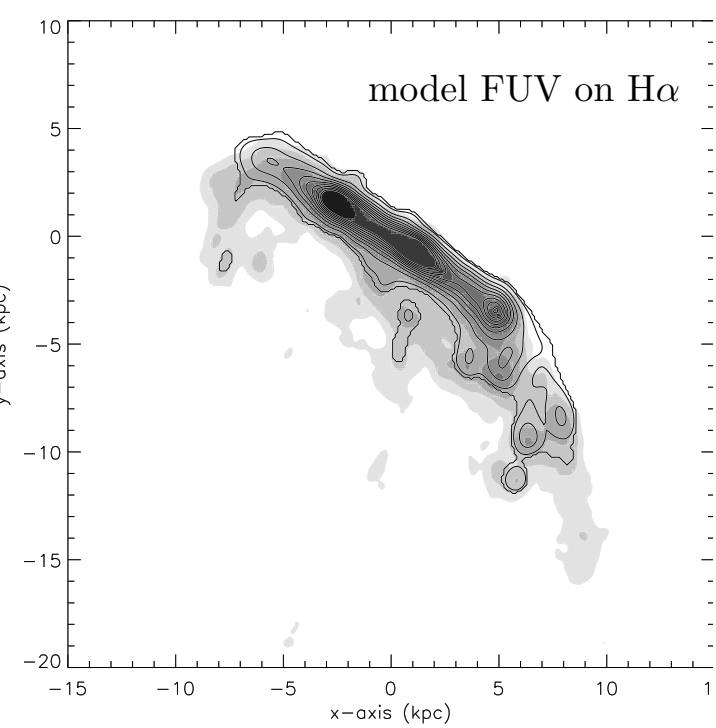

(d)

Fig. 1. NGC 4330. Upper-left panel: VIVA survey HI emission distribution (contours) on the GALEX NUV image. Upper-right panel: GALEX FUV emission distribution (contours) on the VESTIGE continuum-subtracted $\mathrm{H} \alpha+[\mathrm{NII}]$ image. Lower-left panel: model HI emission distribution (contours) on the model extinction-free NUV image. Lower-right panel: model FUV emission distribution (contours) on the model extinction-free $\mathrm{H} \alpha$ image.

surface brightness and can only be detected through deep narrowband observations. Such observations for NGC 4330 have only now become available. Therefore, the Vollmer et al. $(2012 a, b)$ model was upgraded to enable the simulation of diffuse ionized gas stripping.

Within the Virgo Environmental Survey Tracing Ionised Gas Emission (VESTIGE; Boselli et al. 2018), Fossati et al. (2018) detected a low surface brightness $10 \mathrm{kpc} \mathrm{H} \alpha$ tail exhibiting a peculiar filamentary structure. In addition, these authors collected literature photometry in 15 bands from the FUV to the far-infrared (FIR) and VLT FORS2 deep optical long-slit spectroscopy. Using a Monte Carlo code that jointly fits spectroscopy and photometry, they reconstructed the SFHs in apertures along the major axis of NGC 4330. In this way, they found a clear outside-in gradient of the time when the ram-pressure induced star formation quenching started: the outermost radii were stripped about $500 \mathrm{Myr}$ ago, while the stripping reached the inner $5 \mathrm{kpc}$ from the center within the last $100 \mathrm{Myr}$. In addition, Fossati et al. (2018) discovered a low surface brightness $10 \mathrm{kpc}$ tail of ionized gas exhibiting a peculiar filamentary structure (upper-right panel of Fig. 1). The ionized gas tail is associated with the HI tail but extends further to the south.

\section{The dynamical model}

We used the $N$-body code described in Vollmer et al. (2001), which consists of two components: (i) a non-collisional component that simulates the stellar bulge, stellar disk, and the dark halo and (ii) a collisional component that simulates the ISM as an ensemble of gas clouds. A scheme for star formation was implemented, where stars were formed during cloud collisions and then evolved as non-collisional particles. 
Table 1. Total mass, number of collisionless particles $N$, particle mass $M$, and smoothing length $l$ for the different galactic components.

\begin{tabular}{lcccc}
\hline \hline Component & $M_{\text {tot }}\left(M_{\odot}\right)$ & $N$ & $M\left(M_{\odot}\right)$ & $1(\mathrm{pc})$ \\
\hline Halo & $1.7 \times 10^{11}$ & 32768 & $5.0 \times 10^{6}$ & 1200 \\
Bulge & $5.7 \times 10^{9}$ & 16384 & $3.5 \times 10^{5}$ & 180 \\
Disk & $2.8 \times 10^{10}$ & 32768 & $8.7 \times 10^{5}$ & 240 \\
\hline
\end{tabular}

\subsection{Halo, stars, and gas}

The non-collisional component consists of 81920 particles, which simulate the galactic halo, bulge, and disk. The characteristics of the different galactic components are shown in Table 1.

The resulting rotation velocity is $\sim 180 \mathrm{~km} \mathrm{~s}^{-1}$, and the rotation curve becomes flat at a radius of about $5 \mathrm{kpc}$.

We adopted a model where the ISM is simulated as a collisional component, that is, as discrete particles that each possess a mass and a radius and can have inelastic collisions (sticky particles). The advantage of our approach is that ram pressure can be easily included as an additional acceleration on particles that are not protected by other particles (see Vollmer et al. 2001).

The 20000 particles of the collisional component represent gas cloud complexes that evolve in the gravitational potential of the galaxy. The total assumed gas mass is $M_{\text {gas }}^{\text {tot }}=4.3 \times 10^{9} M_{\odot}$, which corresponds to the total neutral gas mass before stripping (i.e., to an HI deficiency of 0 ). A radius is attributed to each particle, which depends on its mass assuming a constant surface density. The normalization of the mass-size relation was taken from Vollmer et al. (2012a). During each cloud-cloud collision, the overlapping parts of the clouds are calculated. Let $b$ be the impact parameter and $r_{1}$ and $r_{2}$ the radii of the larger and smaller clouds. If $r_{1}+r_{2}>b>r_{1}-r_{2}$, the collision can result in fragmentation (high-speed encounter) or mass exchange. If $b<r_{1}-r_{2}$, mass exchange or coalescence (low-speed encounter) can occur. The outcome of the collision is simplified following Wiegel (1994). If the maximum number of gas particles or clouds (20000) is reached, only coalescent or mass-exchanging collisions are allowed. In this way, a cloud mass distribution is naturally produced. The cloud-cloud collisions result in an effective gas viscosity in the disk.

As the galaxy moves through the ICM, its clouds are accelerated by ram pressure $a=\rho_{\mathrm{ICM}} v_{\text {gal }}^{2} / \Sigma$. In addition, the gas clouds are accelerated by the gradients of the gravitational potential $a=-\nabla \phi$. The ISM surface density is assumed to be $\Sigma(r)=$ $(1+\exp (-r / 2 \mathrm{kpc})) \times 10 M_{\odot} \mathrm{pc}^{-2}$. The constant surface density at the outer disk is motivated by the observed saturation of the $\mathrm{HI}$ surface density (e.g., Leroy et al. 2008). The radial dependence takes into account the increased surface density that is due to the presence of molecular gas. Within the galaxy's inertial system, the galaxy's clouds are exposed to a wind coming from a direction opposite to that of the galaxy's motion through the ICM. The temporal ram pressure profile has the form of a Lorentzian, which is realistic for galaxies on highly eccentric orbits within the Virgo cluster (Vollmer et al. 2001). The effect of ram pressure on the clouds is simulated by an additional force on the clouds in the wind direction. Only clouds that are not protected from the wind by other clouds are affected. This results in a finite penetration length of the ICM into the ISM (see Appendix B). Since the gas cannot develop instabilities, the influence of turbulence on the stripped gas is not included in the model. The mixing of the ICM into the ISM is very crudely approximated by the finite penetration length of the ICM into the ISM; in other words, up to this penetration length, the clouds undergo an additional acceleration caused by ram pressure.

\subsection{Star formation}

We assume that the star formation rate (SFR) is proportional to the cloud collision rate. A mass-size relation based on a constant gas surface density is assumed for the collisional particles. The collisions are explicitly calculated for each timestep $\left(\sim 10^{4} \mathrm{yr}\right)$ via the simplified geometrical recipes of Wiegel (1994). We verified that the SFR of an unperturbed galaxy is constant within 1 Gyr.

Vollmer \& Beckert (2003) expanded on an analytical model for a galactic gas disk that considers the warm, cold, and molecular phases of the ISM as a single, turbulent gas. This gas is assumed to be in vertical hydrostatic equilibrium, with the midplane pressure balancing the weight of the gas and the stellar disk. The gas is taken to be clumpy, so that the local density is enhanced relative to the average density of the disk. Using this local density, the free-fall time of an individual gas clump (i.e., the governing timescale for star formation) can be determined. The SFR is used to calculate the rate of energy injection by supernovae. This rate is related to the turbulent velocity dispersion and the driving scale of turbulence. These quantities in turn provide estimates of the clumpiness of gas in the disk (i.e., the contrast between the local and average density) and the rate at which viscosity moves matter inward. Vollmer \& Leroy (2011) applied the model successfully to a sample of local spiral galaxies. Within the framework of the model, the SFR per unit volume is given by

$\dot{\rho}_{*}=\Phi_{\mathrm{V}} \rho t_{\mathrm{ff}, \mathrm{cl}}^{-1}$,

where $\Phi_{\mathrm{V}}$ and $t_{\mathrm{ff}, \mathrm{cl}}^{-1}$ are the volume filling factor and free-fall time of self-gravitating gas clouds, respectively, and $\rho$ is the overall gas density. The volume filling factor is $\Phi_{\mathrm{V}}=n_{\mathrm{cl}} 4 \pi / 3 r_{\mathrm{cl}}^{3}$, where $n_{\mathrm{cl}}$ is the internal number density of clouds and $r_{\mathrm{cl}}$ is the cloud radius. For a self-gravitating cloud, the free-fall time equals the turbulent crossing time $t_{\mathrm{ff}, \mathrm{cl}}=2 r_{\mathrm{cl}} / v_{\text {turb,cl }}$. With the collision time given by $t_{\text {coll }}=\left(n_{\mathrm{cl}} \pi r_{\mathrm{cl}}^{2} l_{\text {turb }}\right)^{-1}$, this leads to $\dot{\rho}_{*}=2 / 3 \rho t_{\text {coll }}^{-1}$. Thus, the SFRs of the two recipes are formally equivalent.

In numerical simulations, the star formation recipe usually involves the gas density $\rho$ and the free-fall time $t_{\mathrm{ff}}=$ $\sqrt{3 \pi /(32 G \rho)}: \dot{\rho}_{*} \propto \rho t_{\mathrm{ff}}^{-1} \propto \rho^{1.5}$. We verified that our star formation recipe based on cloud-cloud collisions leads to the same exponent of the gas density in a simulation of an isolated spiral galaxy. As a consequence, our code reproduces the observed SFR-total gas surface density, SFR-molecular gas surface density, and SFR-stellar surface density relations (Vollmer et al. 2012a,b).

During the simulations, stars are formed in cloud-cloud collisions. In each collision, a collisionless particle is created, which is added to the ensemble of collisional and collisionless particles. The newly created collisionless particles have zero mass (they are test particles) and the positions and velocities of the colliding clouds after the collision. These particles are then evolved passively with the whole system. Since in our sticky-particle scheme there is mass exchange, coalescence, or fragmentation at the end of a collision, the same clouds do not collide infinitely. The local collision rate traces the cloud density and the velocity dispersion of the collisional component.

The information about the time of creation is attached to each newly created star particle. In this way, the $\mathrm{H} \alpha$ emission distribution caused by HII regions can be modeled by the distribution 
of star particles with ages younger than 20 Myr. The UV emission of a star particle in the two GALEX bands is modeled by the UV flux of single stellar population models produced from the STARBURST99 software (Leitherer et al. 1999). The age of the stellar population equals the time since the creation of the star particle. The total UV distribution is then the extinctionfree distribution of the UV emission of the newly created star particles.

\subsection{Diffuse gas stripping}

Our numerical code is not able to treat diffuse gas in a consistent way. For a realistic treatment, 3D hydrodynamical simulations should be adopted. Nevertheless, we can mimic the action of ram pressure on diffuse gas by applying very simple recipes based on the fact that the acceleration by ram pressure is inversely proportional to the gas surface density, which in turn depends on the gas density for a gas cloud of constant mass. For the sake of simplicity, we do not modify the radii of the diffuse gas clouds for the calculation of the cloud-cloud collisions. The diffuse clouds are thus mostly ballistic particles under the influence of a ram-pressure induced acceleration. The recipes only take the gas cooling of the hot phase into account.

We assume that the warm $\left(\sim 10^{4}-10^{5} \mathrm{~K}\right)$ gas clouds become diffuse (i.e., their sizes and volume filling factor increase and their densities and column densities decrease) if they are stripped out of the galactic plane and if their densities fall below the critical density of $n_{\text {crit }}^{\text {warm }}=5 \times 10^{-3} \mathrm{~cm}^{-3}$. We further assume that the first condition is fulfilled if the stellar density at the location of the gas particle is lower than $\rho_{*}^{\text {crit }}=2.5 \times 10^{-4} M_{\odot} \mathrm{pc}^{-3}$. For our model galaxy, this density is reached at a disk height of $\sim 3.5-4 \mathrm{kpc}$.

Once the ionized gas has a high volume filling factor (i.e., it becomes diffuse), its volume and surface densities decrease. The decrease in the surface density is taken into account by considering a gas cloud of a constant mass: for $\rho_{*}<\rho_{*}^{\text {crit }}$, the cloud size is proportional to $n^{-1 / 3}$, the cloud surface density is proportional to $n^{2 / 3}$, and the acceleration caused by ram pressure $a=\rho_{\mathrm{ICM}} v_{\text {gal }}^{2} / \Sigma$ is increased by a factor of $\left(0.044 \mathrm{~cm}^{-3} / n_{\mathrm{ISM}}\right)^{2 / 3}$. This last normalization and the critical densities were chosen such that they led to acceptable results for several observed galaxies undergoing ram pressure stripping. The critical stellar density is motivated by the fact that a significant change in the ISM properties only occurs once the ISM has entirely left the galactic disk. It turns out that this condition is necessary to avoid excessive gas stripping. The gas and stellar densities are calculated via the 50 nearest neighboring particles.

The hot $\left(>10^{6} \mathrm{~K}\right)$ diffuse gas is taken into account in the following way: We assume that once the stripped gas has left the galactic disk, it mixes with the ambient ICM. The initial temperature of the ISM is $T_{\text {ISM }}=10^{4} \mathrm{~K}$. If (i) the gas density falls below the critical value of $n_{\text {crit }}^{\text {hot }}=5 \times 10^{-4} \mathrm{~cm}^{-3}$, (ii) the stellar density is below $\rho_{*}^{\text {crit }}$, and (iii) the ISM temperature is below $9 \times 10^{6} \mathrm{~K}$, ICM-ISM mixing begins to take place. On the other hand, if the stellar density exceeds its critical value (i.e., the gas is located within or close to the galactic disk), then the gas is assumed to cool rapidly and its temperature is set to $T_{\mathrm{ISM}}=10^{5} \mathrm{~K}^{1}$. We assume that mixing occurs instantaneously and raises the temperature of the mixed gas clouds to

\footnotetext{
1 A temperature of $T_{\text {ISM }}=10^{4} \mathrm{~K}$ does not change the results of the simulations.
}

$$
T=\frac{n_{\mathrm{ICM}} 3 \times 10^{7} \mathrm{~K}+\sqrt{n_{\mathrm{ICM}} n_{\mathrm{ISM}}} T_{\mathrm{ISM}}}{n_{\mathrm{ICM}}+\sqrt{n_{\mathrm{ICM}} n_{\mathrm{ISM}}}}=9 \times 10^{6} \mathrm{~K},
$$

where $n_{\mathrm{ICM}}$ is the density of the mixed ICM, which is calculated for each particle. If an exceedingly high mixed ICM density $\left(n_{\mathrm{ICM}}>2 \times 10^{-3} \mathrm{~cm}^{-3}\right)$ is needed to heat the gas cloud to $9 \times 10^{6} \mathrm{~K}$, ICM-ISM heating is not allowed. The temperature of the mixed hot gas clouds $T_{\text {ISM }}$, if not heated, decreases with a cooling timescale of $t_{\text {cool }}=1.5 \times 10^{7} n_{\text {ISM }}^{-1} \mathrm{yr}$, where the ISM density $n_{\mathrm{ISM}}$ is given in $\mathrm{cm}^{-3}$. Once mixed, the gas cloud is not allowed to cool below a temperature of $10^{6} \mathrm{~K}^{2}$. This algorithm ensures that stripped gas below the critical density $n_{\text {crit }}$ is kept at a temperature of $9 \times 10^{6} \mathrm{~K}$, whereas denser gas can cool to a temperature of $10^{6} \mathrm{~K}$.

For the stripped hot gas clouds $\left(>10^{6} \mathrm{~K}\right)$, we modified the equation of motion by adding the acceleration caused by the gas pressure gradient $a=-\nabla p_{\text {ISM }} / \rho_{\text {ISM }}$ using a Smoothed-particle hydrodynamics (SPH) formalism. For the sake of simplicity, an isothermal stripped ISM with a sound speed of $c_{\mathrm{s}}=235 \mathrm{~km} \mathrm{~s}^{-1}$ is assumed for this purpose. We thus do not solve an explicit energy equation and the calculation of hydrodynamic effects is approximate. Its main purpose is to keep the hot diffuse ISM from clumping.

For the calculation of the acceleration caused by ram pressure $a=\rho_{\mathrm{ICM}} v_{\text {gal }}^{2} / \Sigma$, the acceleration is further increased by a heuristic factor of ten if the ISM temperature exceeds $10^{5} \mathrm{~K}$ and the stellar density is below $\rho_{*}=2.5 \times 10^{-4} M_{\odot} \mathrm{pc}^{-3}$. We note that without the inclusion of hydrodynamical effects, thin gas filaments cannot be produced by our simple model. The diffuse gas is assumed to emit in the $\mathrm{H} \alpha$ line if its temperature is below $10^{6} \mathrm{~K}$.

\subsection{Parameters of the ram pressure stripping event}

Following Vollmer et al. (2001), we used a Lorentzian profile for the time evolution of ram pressure stripping:

$p_{\mathrm{rp}}=p_{\mathrm{max}} \frac{t_{\mathrm{HW}}^{2}}{\left(t-t_{\text {peak }}\right)^{2}+t_{\mathrm{HW}}^{2}}$,

where $p_{\max }$ is the maximum ram pressure occurring at the galaxy's closest passage to the cluster center, $t_{\mathrm{HW}}$ is the width of the profile, and $t_{\text {peak }}$ is the time of peak ram pressure. The simulations were calculated from $t=0$ to $t=$ 800 Myr. We set $p_{\max }=5000,6000,10000 \mathrm{~cm}^{-3}\left(\mathrm{~km} \mathrm{~s}^{-1}\right)^{2}$ and $t_{\mathrm{HW}}=100,200,300$ Myr. Following Nehlig et al. (2016) and Vollmer et al. (2018), we investigated the influence of galactic structure (i.e., the position of spiral arms) on the results of ram pressure stripping by varying the time of peak ram pressure between $t_{\text {peak }}=570 \mathrm{Myr}$ and $690 \mathrm{Myr}$. The ram pressure profiles are specified in Table 2 and shown in Fig. 2. The parameters of the Vollmer et al. $(2012 \mathrm{a}, \mathrm{b})$ model were $p_{\max }=$ $5000 \mathrm{~cm}^{-3}\left(\mathrm{~km} \mathrm{~s}^{-1}\right)^{2}$ and $t_{\mathrm{HW}}=100 \mathrm{Myr}$.

We adopted the Vollmer et al. (2012a,b) values for the angle between the ram pressure wind and the disk plane $\left(75^{\circ}\right)$. The inclination of the model galaxy is $i=90^{\circ}$ to the line-of-sight.

2 A temperature limit of $T_{\text {ISM }}=10^{5} \mathrm{~K}$ does not change the results of the simulations because the cooling times of the X-ray emitting gas are on the order of several gigayears. Cooling of the hot gas does not occur in these simulations. 
Table 2. Model ram pressure stripping profiles.

\begin{tabular}{lccc}
\hline \hline Model & $\begin{array}{c}\text { Amplitude } p_{\text {max }} \\
\left(\mathrm{cm}^{-3}\left(\mathrm{~km} \mathrm{~s}^{-1}\right)^{2}\right)\end{array}$ & $\begin{array}{c}\text { Width } t_{\text {HW }} \\
(\mathrm{Myr})\end{array}$ & $\begin{array}{c}\text { Peak time } t_{\text {peak }} \\
(\mathrm{Myr})\end{array}$ \\
\hline 1, 1new & 10000 & 200 & 600 \\
1a, 1anew & 10000 & 200 & 570 \\
1b, 1bnew & 10000 & 200 & 630 \\
1c, 1cnew & 10000 & 200 & 660 \\
1d, 1dnew & 10000 & 200 & 690 \\
2, 2new & 5000 & 300 & 600 \\
2a, 2anew & 5000 & 300 & 570 \\
2b, 2bnew & 5000 & 300 & 630 \\
2c, 2cnew & 5000 & 300 & 660 \\
2d, 2dnew & 5000 & 300 & 690 \\
3, 3new & 10000 & 300 & 600 \\
3a, 3anew & 10000 & 300 & 570 \\
3b, 3bnew & 10000 & 300 & 630 \\
3c, 3cnew & 10000 & 300 & 660 \\
3d, 3dnew & 10000 & 300 & 690 \\
4, 4new & 6000 & 200 & 600 \\
4a, 4anew & 6000 & 200 & 570 \\
4b, 4bnew & 6000 & 200 & 630 \\
4c, 4cnew & 6000 & 200 & 660 \\
4d, 4dnew & 6000 & 200 & 690 \\
5, 5new & 10000 & 100 & 600 \\
5a, 5anew & 10000 & 100 & 570 \\
5b, 5bnew & 10000 & 100 & 630 \\
5c, 5cnew & 10000 & 100 & 660 \\
5d, 5dnew & 10000 & 100 & 690 \\
\hline
\end{tabular}

Notes. Nomenclature: Models $\mathrm{Na}$ : without a diffuse ISM component. Models Nanew: with a diffuse ISM component.

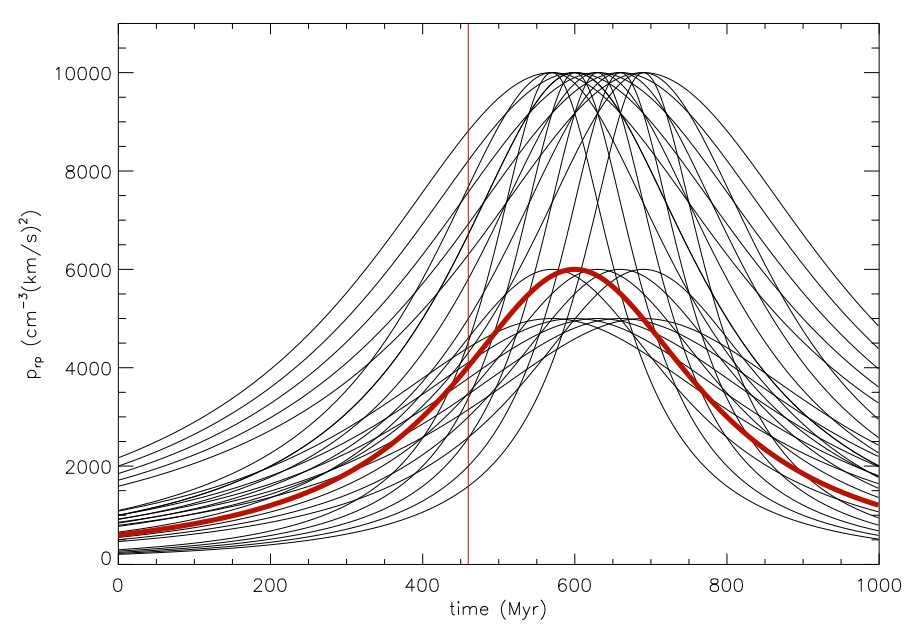

Fig. 2. Model ram pressure profiles. The thick red line shows the best-fit model, and the vertical red line shows the timestep of the best-fit model.

\section{Observations and model fitting}

The deep optical spectrum was obtained with the FOcal Reducer and low dispersion Spectrograph (FORS2; Appenzeller et al. 1998) mounted on Unit Telescope 1 of the ESO VLT. With a length of $6.8^{\prime}$, the FORS2 slit covered the full extent of the galaxy. A slit aperture of $1.3^{\prime \prime}$ was used to optimize the covered area without compromising the spectral resolution of $R \sim 1000$. We obtained six exposures of $900 \mathrm{~s}$ each using the $600 \mathrm{~B}+22$ grism, which covers the wavelength range $3300-6210 \AA$, and three exposures of $900 \mathrm{~s}$ each using the $600 \mathrm{RI}+19$ grism, which covers the wavelength range 5120$8450 \AA$. The deep continuum-subtracted $\mathrm{H} \alpha+[\mathrm{NII}]$ observations were obtained within the VESTIGE survey (Boselli et al. 2018) using MegaCam at the Canada-French-Hawaii Telescope (CFHT) with the narrowband filter (MP9603) and the broadband $r$ filter (MP9602). The ancillary GALEX, CFHT, Calar Alto $2.2 \mathrm{~m}$ telescope, Spitzer, and Herschel photometric data are described in Fossati et al. (2018). In addition, for the comparison with our models, we used VIVA survey HI and $20 \mathrm{~cm}$ continuum data (Chung et al. 2009) and $6 \mathrm{~cm}$ radio continuum data from Vollmer et al. (2013).

Fossati et al. (2018) reconstructed the quenching histories of NGC 4330 in various regions along the major axis by means of stellar population fitting. They performed a joint fitting of spectra and photometry using Monte Carlo techniques to optimally explore the parameter space. The emission of the galactic disk was divided into 13 apertures along the major axis of NGC 4330, as shown in Fig. 3.

Aperture photometry was obtained by summing the calibrated flux values in each pixel that contributes to a given aperture minus the background value in the same aperture. To estimate the background, each aperture was randomly placed in empty regions of the images and the sum of the counts was taken. This procedure was repeated 1000 times and the median value was adopted as the best estimator for the background value. For the UV, optical, and near-infrared (NIR) bands, the measured fluxes were corrected for the Galactic attenuation. The spectra from the FORS2 reduced 2D spectral images were extracted by summing the flux in the spatial pixels contributing to each region along the slit direction.

For the reconstruction of the quenching history of each radial bin, a model of its unperturbed radial SFH is needed. For this purpose, Fossati et al. (2018) adopted the multizone models for the chemical and spectrophotometric unperturbed disk evolution originally presented in Boissier \& Prantzos (2000) and later updated by Muñoz-Mateos et al. (2011). The model was projected to fit the high inclination of the disk of NGC 4330, and the SFHs were derived in each of the apertures. Fossati et al. (2018) further modified the unperturbed SFHs by introducing an exponential decline of the SFR to parametrize the quenching event.

Parametric models to the spectrophotometric data were produced using MULTINEST (Feroz \& Hobson 2008; Feroz et al. 2009; Feroz \& Skilling 2013). A grid of stellar spectra was constructed using the quenched SFHs coupled to Bruzual \& Charlot (2003) high-resolution models. Nebular emission lines were added to the stellar template. To do so, Fossati et al. (2018) first computed the number of Lyman continuum $(\lambda<912 \AA)$ photons $Q_{\mathrm{H}_{0}}$ for a given stellar spectrum. They then assumed that all the ionizing radiation is absorbed by the gas and that the ionizing photons do not contribute to the heating of dust. However, they modeled the uncertainty in the conversion of $Q_{\mathrm{H}_{0}}$ into emission line flux by adding a nuisance parameter $\left(\mathrm{Ly}_{\text {scale }}\right)$ in their Monte Carlo Spectro-Photometric Fitter (MC-SPF) code. This parameter also takes into account differential aperture effects between the $1.3^{\prime \prime}$ spectral slit and the larger photometric apertures. A double (Calzetti et al. 2000) attenuation law was assumed to include the effects of dust attenuation on the stellar and nebular emission line spectrum. Fossati et al. (2018) coupled the stellar spectrum with dust emission models from the mid-infrared to the FIR by means of an energy balance between the stellar flux absorbed by the dust and reemitted in the infrared. They jointly fit the photometric data points and the FORS2 spectra at their native 

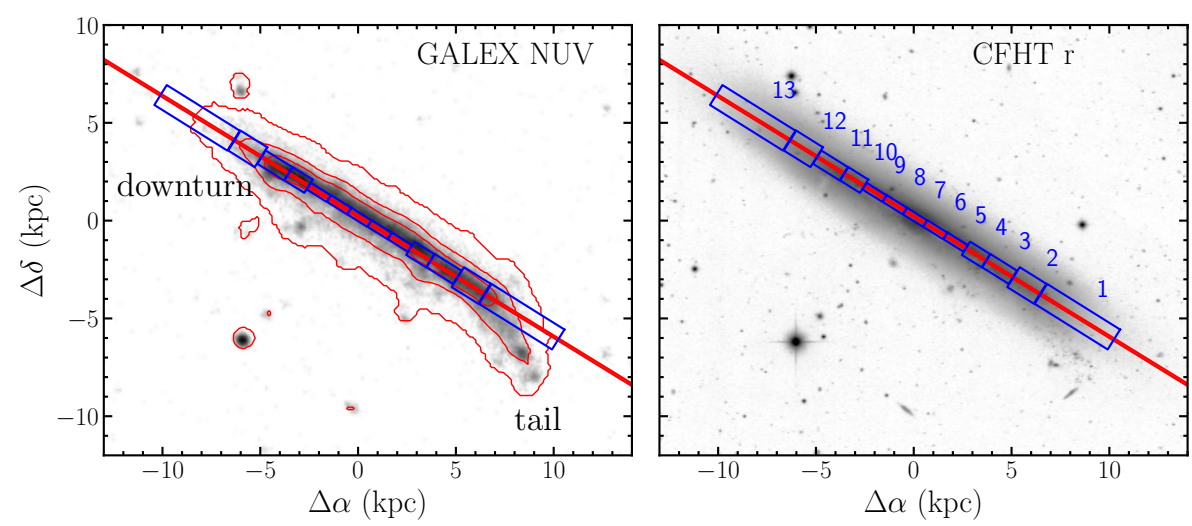

Fig. 3. NGC 4330. Left panel: VLT slit position on the GALEX NUV image. Right panel: VLT slit position on the CFHT $r$ band image adapted from Fossati et al. (2018). The downturn region includes slit regions 11 to 13 , and the tail region includes slit regions $1-3$. The downturn plus tail region includes slit regions $1-3$ and $11-13$. resolution. The multidimensional likelihood space was sampled by using PYMULTINEST (Buchner et al. 2014), a python wrapper for the MULTINEST code. For direct comparison with the results obtained from our model SFHs, we reproduce the results of Fossati et al. (2018) in Figs. A.4-A.9.

For our purposes, we used the modeling of Fossati et al. (2018) to obtain the synthetic photometry and the optical spectra for our model SFHs. The model photometry and spectrum is then compared to observations using MULTINEST and the total evidence is calculated. The goodness is evaluated using the Bayesian evidence. This value is formally defined as the integral of the likelihood of a given model over the entire parameter space. When two models are compared, their goodness in describing the data can be summarized in the ratio of their evidence (or the difference in log space). The Bayesian evidence automatically penalizes models with more free parameters, thus optimally highlighting the best model even in the event that the models under considerations have a different number of free parameters. In our case, these free parameters are the temporal ram pressure profile, the chosen timestep, and the inclusion of diffuse gas stripping. The variation of the parameters that are used to compare the model to the data (stellar libraries, the attenuation model, and the $\mathrm{Ly}_{\text {scale }}$ parameter) can change the goodnesses and thus the order of the preferred models. A better model yields a higher goodness.

\section{Results}

We calculated the model photometry and spectrum for all timesteps $(\Delta t=10 \mathrm{Myr})$ of the 50 simulations presented in Table 2 with (format $N a$ new) and without (format $N a$ ) a diffuse gas component. In addition, we decided to handle the Ly scale nuisance parameter in two different ways: (i) We set it to the fixed values found by Fossati et al. (2018), and (ii) we treated it as a free parameter and kept the value that led to the best fit to observations. The distributions of the total goodnesses for all model timesteps are shown in Fig. C.1. The distributions show a tail to high values containing about 10-20 timesteps. The resulting total goodnesses are presented in Table 3. The last number of the model names is the simulation timestep $N$. The time to peak ram pressure is $((N-1) \times 10)-t_{\text {peak }} \mathrm{Myr}$, where $t_{\text {peak }}$ is given in Table 2.

We then searched for the 12 timesteps with the highest goodnesses for the downturn, tail, and total (downturn plus tail) regions. These different regions are generally not best fit by a single timestep (Table 4). Figure C.2, compared to Fig. A.6, gives an impression of the meaning of the total goodness $g$ : The model as well as the observed spectra and spectral energy distri- butions (SEDs) of region 3 for two timesteps (models 4new_47 and 1cnew_46), with a goodness difference of $\Delta g \sim 200$, show that the FUV and NUV fluxes are significantly better reproduced by model 4new_47. We therefore presume that goodness differences in excess of $\Delta g \gtrsim 200$ are significant.

All of our total model goodnesses are smaller than that of the parametric (Fossati et al. 2018) model. This is expected because the parametric model had a higher degree of freedom. The models with a diffuse stripped ISM lead to somewhat higher goodnesses than those without a diffuse stripped ISM. Moreover, models with a variable Ly scale $_{\text {parameter sys- }}$ tematically yield higher goodnesses thanks to the physical relevance of this parameter in reproducing the observed emission lines in the spectra. This is true for the downturn, tail, and total regions.

Model 4new_47 with diffuse gas stripping has the highest total goodness of all the tested models (Table 3). Given that a goodness difference of $\Delta g \gtrsim 200$ is significant, models $1 \mathrm{cnew} 47$ (diffuse gas stripping), 4b_67, and 2b_80 are also acceptable amongst the models with a variable $\mathrm{Ly}_{\text {scale }}$ parameter. For a constant $\mathrm{Ly}_{\text {scale }}$ parameter, only model $2 \mathrm{~b} \_80$ is acceptable.

If we look at the goodnesses of the downturn and tail regions separately (Table 4), the results change: In the case of a variable $\mathrm{Ly}_{\text {scale }}$ parameter, all 12 best-fit models of the downturn region are acceptable whether or not diffuse gas stripping is included. For the tail region, all models without diffuse gas stripping are acceptable. If the stripping of diffuse gas is included in the simulations, only the first three models are acceptable. In the case of a constant $\mathrm{Ly}_{\text {scale }}$ parameter, the four and two best-fit models are acceptable whether or not diffuse gas stripping is included. In the tail region, only the eight best-fit models without diffuse gas stripping are acceptable.

We conclude that the best-fit models based on the total goodness are pre-peak ram pressure models if diffuse gas stripping is included, otherwise the preferred timesteps are post-peak ram pressure (see Table 2).

\subsection{The NUV and HI morphologies}

The observed HI emission stems from warm ( $~ 8000 \mathrm{~K})$ gas with densities $\gtrsim 1 \mathrm{~cm}^{-3}$. The NUV is emitted by the photospheres of massive hot stars. It traces the star formation on timescales $<300$ Myr.

The 48 simulation snapshots of Table 3 yield the highest total goodnesses for each kind of simulation (with or without diffuse gas stripping, with a variable or constant $\mathrm{Ly}_{\text {scale }}$ parameter). Models with total goodnesses that exceed $g=32000$ for 
Table 3. Model fitting results.

\begin{tabular}{|c|c|c|c|c|c|c|c|c|}
\hline $\begin{array}{l}\text { Downturn+tail } \\
\text { model }\end{array}$ & $\begin{array}{c}\text { Total } \\
\text { goodness }\end{array}$ & $\begin{array}{c}\text { Down } \\
\text { HI }\end{array}$ & $\begin{array}{l}\text { Turn } \\
\text { NUV }\end{array}$ & $\mathrm{H} \alpha$ & $\begin{array}{c}\text { Tail } \\
\text { HI }\end{array}$ & NUV & $\mathrm{H} \alpha$ & $\begin{array}{c}\text { Time to } t_{\text {peak }} \\
(\mathrm{Myr})\end{array}$ \\
\hline Fossati et al. & 32621 & & & & & & & \\
\hline With diffuse ISM & Component & & & & & & & \\
\hline Variable $\mathrm{Ly}_{\text {scale }}$ & Parameter & & & & & & & \\
\hline 4new_47 & 32209 & + & $\sim$ & + & + & $\sim$ & + & -140 \\
\hline 1cnew_47 & 32003 & - & + & $\sim$ & + & + & $\sim$ & -200 \\
\hline 3dnew_47 & 31719 & - & - & - & - & - & - & -230 \\
\hline 4anew_47 & 31658 & - & $\sim$ & $\sim$ & + & $\sim$ & - & -110 \\
\hline 2anew_64 & 31647 & - & - & - & $\sim$ & - & - & +60 \\
\hline 2anew_65 & 31605 & - & - & $\sim$ & - & - & - & +70 \\
\hline 4new_59 & 31589 & - & - & - & - & $\sim$ & - & -20 \\
\hline 4anew_60 & 31557 & - & - & - & - & - & - & +20 \\
\hline 4new_61 & 31541 & $\sim$ & - & + & + & $\sim$ & - & 0 \\
\hline $1 \mathrm{cnew}$-46 & 31467 & - & - & - & $\sim$ & + & $\sim$ & -210 \\
\hline 1dnew_47 & 31433 & - & - & - & + & + & $\sim$ & -230 \\
\hline 4anew_59 & 31423 & - & - & $\sim$ & - & $\sim$ & - & +10 \\
\hline Constant Ly $\mathrm{y}_{\text {scale }}$ & Parameter & & & & & & & \\
\hline 4anew_60 & 31248 & $\sim$ & - & + & $\sim$ & $\sim$ & - & +20 \\
\hline 2anew_65 & 30771 & - & - & $\sim$ & - & - & - & +70 \\
\hline 4dnew_58 & 30373 & + & - & $\sim$ & + & $\sim$ & - & -120 \\
\hline 4dnew_59 & 30240 & + & - & - & + & $\sim$ & - & -110 \\
\hline 4cnew_57 & 29978 & - & - & + & $\sim$ & $\sim$ & - & -100 \\
\hline 4dnew_57 & 29969 & + & - & $\sim$ & + & $\sim$ & - & -130 \\
\hline 4new_47 & 29961 & + & $\sim$ & + & + & $\sim$ & + & -140 \\
\hline 2anew_56 & 29898 & - & - & - & - & - & - & -20 \\
\hline 2anew_55 & 29880 & - & - & $\sim$ & - & - & $\sim$ & -30 \\
\hline 2anew_64 & 29868 & - & - & $\sim$ & $\sim$ & - & - & +60 \\
\hline 2bnew_73 & 29845 & - & - & $\sim$ & $\sim$ & - & $\sim$ & +90 \\
\hline 4cnew_56 & 29575 & $\sim$ & - & + & - & - & - & -110 \\
\hline Without diffuse ISM & Component & & & & & & & \\
\hline Variable $\mathrm{Ly}_{\text {scale }}$ & Parameter & & & & & & & \\
\hline 4b_67 & 32103 & - & - & + & $\sim$ & $\sim$ & - & +30 \\
\hline $2 b \_80$ & 32004 & - & $\sim$ & + & - & - & $\sim$ & +160 \\
\hline $3 c \_47$ & 31904 & - & $\sim$ & + & $\sim$ & $\sim$ & - & -200 \\
\hline $4 b \_68$ & 31897 & - & - & - & $\sim$ & $\sim$ & - & +40 \\
\hline $2 d_{-} 70$ & 31816 & $\sim$ & $\sim$ & + & $\sim$ & $\sim$ & + & 0 \\
\hline $3 \_47$ & 31795 & $\sim$ & + & $\sim$ & $\sim$ & $\sim$ & $\sim$ & -140 \\
\hline $4 \mathrm{c} \_70$ & 31795 & - & - & $\sim$ & $\sim$ & - & $\sim$ & +30 \\
\hline $3 b \_47$ & 31760 & - & $\sim$ & + & $\sim$ & $\sim$ & - & -170 \\
\hline $4 d_{-} 70$ & 31708 & + & + & $\sim$ & $\sim$ & $\sim$ & + & 0 \\
\hline $2 a \_68$ & 31699 & - & $\sim$ & - & $\sim$ & $\sim$ & - & +70 \\
\hline $2 c \_80$ & 31695 & $\sim$ & - & - & - & - & - & +130 \\
\hline $3 \mathrm{~d} \_48$ & 31671 & - & - & + & + & $\sim$ & - & -220 \\
\hline Constant $\mathrm{Ly}_{\text {scale }}$ & Parameter & & & & & & & \\
\hline 2b_80 & 31854 & - & - & + & - & $\sim$ & - & +160 \\
\hline 3d_48 & 31263 & - & - & + & + & $\sim$ & - & -220 \\
\hline 2d_77 & 30802 & + & - & + & + & + & - & +70 \\
\hline 3c_48 & 30689 & - & - & + & + & $\sim$ & - & -190 \\
\hline 2d_74 & 30685 & - & - & + & $\sim$ & $\sim$ & - & +40 \\
\hline 3d_57 & 30455 & $\sim$ & - & + & - & + & - & -130 \\
\hline 2d_70 & 30386 & $\sim$ & $\sim$ & + & $\sim$ & $\sim$ & + & 0 \\
\hline 2b_65 & 30303 & - & - & - & $\sim$ & $\sim$ & - & +10 \\
\hline $2 \mathrm{~d} \_66$ & 30126 & $\sim$ & - & $\sim$ & $\sim$ & $\sim$ & $\sim$ & -40 \\
\hline $2 \mathrm{c} \_80$ & 30062 & $\sim$ & - & - & - & - & - & +130 \\
\hline $4 b \_67$ & 30047 & - & - & + & $\sim$ & $\sim$ & - & +30 \\
\hline 3d_56 & 29908 & - & - & - & - & - & - & -140 \\
\hline
\end{tabular}

Notes. The time to peak ram pressure is $((N-1) \times 10)-t_{\text {peak }}$ Myr, where $N$ is the number of the timestep (last number of the model names). The quality assessment of the reproduction of the observational characteristics is based on the joint fit of the SEDs and optical spectra. + reproduced; $\sim$ : approximately reproduced; - : not reproduced Models $N a$ : without diffuse ISM component. Models Nnewa: with diffuse ISM component. The different regions (downturn, tail, and downturn+tail) are defined in Fig. 3.

a variable $\mathrm{Ly}_{\text {scale }}$ parameter (4new_47, 1cnew_47, 4b_67, and 2b_80) and $g=31650$ for a constant $\mathrm{Ly}_{\text {scale }}$ parameter (2b_80) are acceptable fits to the spectrophotometric observations. However, the observed HI, NUV, and $\mathrm{H} \alpha$ morphologies also have to be reproduced by the model snapshots. To assess the quality of the fits, we compared the model and observed morphologies of the downturn and tail regions by eye. The results are presented
Table 4. Model fitting results.

\begin{tabular}{|c|c|c|c|c|c|}
\hline Downturn & $\begin{array}{l}\Delta t_{\text {peak }} \\
(\mathrm{Myr})\end{array}$ & Goodness & Tail & $\begin{array}{l}\Delta t_{\text {peak }} \\
(\mathrm{Myr})\end{array}$ & Goodness \\
\hline With diffuse ISM & Component & & & & \\
\hline Variable $\mathrm{Ly}_{\text {scale }}$ & Parameter & & & & \\
\hline 2bnew_63 & -10 & 15120 & 5dnew_58 & -120 & 17351 \\
\hline 3dnew_51 & -190 & 15109 & 4new_47 & -140 & 17268 \\
\hline 2dnew_61 & -90 & 15082 & 5cnew_57 & -100 & 17261 \\
\hline 2dnew_62 & -80 & 15071 & 5cnew_58 & -90 & 17254 \\
\hline 4anew_60 & +20 & 15066 & 4cnew_57 & -100 & 17221 \\
\hline 1bnew_48 & -160 & 15053 & 4cnew_56 & -110 & 17214 \\
\hline 2new_76 & +150 & 15049 & 5cnew_66 & -10 & 17196 \\
\hline $1 \mathrm{cnew} \_47$ & -200 & 15048 & 5bnew_63 & -10 & 17196 \\
\hline 2anew_64 & +60 & 15047 & $1 \mathrm{cnew}$-46 & -210 & 17175 \\
\hline 2bnew_62 & -20 & 15045 & 5bnew_69 & +50 & 17174 \\
\hline 1dnew_55 & -130 & 15043 & 1bnew_47 & -170 & 17159 \\
\hline 2anew_42 & -160 & 15039 & 4dnew_58 & -120 & 17132 \\
\hline Constant Ly scale $_{\text {e }}$ & Parameter & & & & \\
\hline 4anew_60 & +20 & 15039 & 5cnew_58 & -90 & 17135 \\
\hline 2dnew_62 & -80 & 15036 & 4dnew_58 & -120 & 17113 \\
\hline 1bnew_48 & -160 & 14917 & 4cnew_56 & -110 & 17029 \\
\hline 1dnew_55 & -150 & 14875 & 5cnew_65 & -20 & 17023 \\
\hline 1dnew_41 & -290 & 14857 & 4new_61 & 0 & 17022 \\
\hline 1cnew_54 & -130 & 14854 & 5cnew_57 & -100 & 17001 \\
\hline 2cnew_75 & +80 & 14814 & 4anew_47 & -110 & 16968 \\
\hline 2anew_47 & -110 & 14812 & 5dnew_76 & +60 & 16950 \\
\hline 2cnew_78 & +110 & 14768 & 1dnew_47 & -230 & 16903 \\
\hline 2cnew_76 & +90 & 14746 & 5new_62 & +10 & 16900 \\
\hline 2cnew_66 & -10 & 14727 & 5bnew_63 & -10 & 16823 \\
\hline 2dnew_61 & -90 & 14720 & 4dnew_57 & -130 & 16820 \\
\hline Without diffuse ISM & Component & & & & \\
\hline Variable $\mathrm{Ly}_{\text {scale }}$ & Parameter & & & & \\
\hline $3 \mathrm{~d} \_44$ & -260 & 15074 & $2 \_58$ & -30 & 17461 \\
\hline $2 b \_80$ & +160 & 15030 & $4 d_{-} 70$ & 0 & 17408 \\
\hline $3 \mathrm{~d}-41$ & -290 & 15012 & $2 \_57$ & -40 & 17398 \\
\hline $1 \mathrm{c} 42$ & -250 & 15006 & 1a 48 & -100 & 17390 \\
\hline $1 \_43$ & -180 & 14983 & $2 a \_58$ & 0 & 17362 \\
\hline $1 \mathrm{~b} \_42$ & -220 & 14973 & $3 b \_48$ & -160 & 17357 \\
\hline $3 \_41$ & -200 & 14962 & $2 \mathrm{~d} \_66$ & -40 & 17356 \\
\hline $2 \mathrm{a} \_66$ & +80 & 14961 & 4_54 & -70 & 17355 \\
\hline $4 c \_68$ & +10 & 14960 & $2 \_56$ & -50 & 17355 \\
\hline $2 a \_65$ & +70 & 14958 & $2 \mathrm{c} \_74$ & +70 & 17333 \\
\hline $3 \mathrm{~d} \_42$ & -280 & 14954 & $2 b \_57$ & -70 & 17324 \\
\hline $3 \mathrm{~d} \_43$ & -270 & 14950 & 3_47 & -140 & 17324 \\
\hline Constant Ly $\mathrm{y}_{\text {scale }}$ & Parameter & & & & \\
\hline $2 b \_80$ & +160 & 15008 & $2 a \_58$ & 0 & 17360 \\
\hline $3 \mathrm{~d} \_44$ & -260 & 14860 & $2 d_{-} 66$ & -40 & 17339 \\
\hline $2 a \_63$ & +50 & 14733 & $2 d_{-} 70$ & 0 & 17294 \\
\hline 2a_66 & +80 & 14717 & 3_47 & -140 & 17285 \\
\hline $2 \mathrm{a} \_65$ & +70 & 14599 & $1 \bar{d} \_58$ & -120 & 17230 \\
\hline $1 \mathrm{~d} \_41$ & -290 & 14565 & $2 c_{-} 72$ & +50 & 17190 \\
\hline $3 c \_46$ & -210 & 14484 & 3d_58 & -120 & 17185 \\
\hline 2c_67 & 0 & 14448 & 2d_77 & +70 & 17174 \\
\hline 2c_80 & +130 & 14275 & 4d_70 & 0 & 17157 \\
\hline 1c_41 & -260 & 14245 & 4_66 & +50 & 17118 \\
\hline 2b_79 & +150 & 14244 & $2 \_58$ & -30 & 17111 \\
\hline 2c_68 & +10 & 14211 & 3d_48 & -220 & 17094 \\
\hline
\end{tabular}

Notes. The time to peak ram pressure is $\Delta t_{\text {peak }}=((N-1) \times 10)-$ $t_{\text {peak }} \mathrm{Myr}$, where $N$ is the number of the timestep (last number of the model names). Models Na: without diffuse ISM component. Models $N$ new $a$ : with diffuse ISM component. The different regions (downturn, tail) are defined in Fig. 3.

in Table 3. The key features used for the quality assessment are: HI: truncated disk, extraplanar gas south of the galactic disk, and southwestern tail; NUV: disk emission extending farther than the HI emission and southwestern tail offset from the HI tail; $\mathrm{H} \alpha$ : truncated disk, southwestern tail offset from the NUV tail, and low surface brightness north-south filaments. The "+" sign indicates it is reproduced, the " $\sim$ " indicates it is approximately reproduced, and "-" indicates it is not reproduced.

The model NUV and HI distributions of the six model snapshots with the highest goodnesses from the simulations with a 
diffuse ISM component and a variable Ly scale $_{\text {scale parameter }}$ are presented in Fig. 4. They can be directly compared to the upper-left panel of Fig. 1.

The observed truncation radius of the HI disk of $7.5 \mathrm{kpc}$ as well as its symmetry along the major axis are well reproduced by all model snapshots. The observed strong asymmetry of the HI distribution along the minor axis with a significant amount of gas pushed by ram pressure downwind of the major axis is also reproduced by all model snapshots. The observed southwestern tail is present in all model snapshots. However, the gas tails of models 4new_47, 1cnew_47, 3dnew_47, and 4anew_47 are somewhat more extended than the observed gas tail. Moreover, the gas distribution of the eastern downturn region of all model snapshots are more extended in the downwind direction than is observed. The observed gas distribution of the downturn region is best reproduced by model 4new_47.

In the downturn region, the observed NUV distribution is more extended along the disk than the HI distribution. This feature is reproduced by all model snapshots. However, the extent of all six model NUV disks are smaller than the observed one. In addition, the NUV emission of all model snapshots is extended in the downwind direction. While the model NUV downturn filaments are perpendicular to the galactic disk in models 1cnew_47, 4anew_47, 2anew_64, and 2anew_65, they are further bent toward the galactic disk in models 4new_47 and 3dnew_47. In this respect, the latter two model snapshots are most similar to observations.

We observe an interesting feature in the NUV distribution of models 2anew_64 and 2anew_65: an extension in the upwind direction on the northwestern side of the galactic disk. This extent is caused by star created in the UV tail that has the time to rotate into the downturn region. Since their angular momentum is that of the tail gas, these stars rotate in a plane that is tilted with respect to the galactic plane, leading to a position angle opposite to that of the tail in the downturn region. Because these stars need to have the time to fulfill about half of a galactic rotation, this effect occurs in simulation snapshots more than several 10 Myr after peak ram pressure. These model snapshots can thus be excluded. The observed bending of the southwestern NUV tail is reproduced by all model snapshots. Its observed offset with respect to the HI tail is only present in model 1cnew_47. We conclude that the observed HI and NUV morphologies are best reproduced by model 4new_47, followed by model 1cnew_47.

To demonstrate the effect of diffuse gas stripping on the model HI and NUV distribution, we show the same model snapshots as in Fig. 4 for the simulation without diffuse gas stripping in Fig. 5. The resulting HI and NUV distributions of all model snapshots are more extended than those of the snapshots of the corresponding models with diffuse gas stripping and observations. Indeed, the snapshots with the highest goodnesses for the models without diffuse gas stripping are not the same as those presented in Figs. 4 and 5 (see Table 3).

The snapshots with the highest goodnesses for the models without diffuse gas are presented in Fig. 6. All model with high snapshot numbers (4b_67, 2b_80,4b_68, and 2d_70) show extensions in the upwind direction on the northwestern side of the galactic disk and are therefore excluded. The HI and NUV tails of the two other models, 3c_47 and 3_47, are less bent than the best-fit models with diffuse gas stripping (4new_47 and 1cnew_47). In addition, extended HI tails in the downturn regions of 3c_47 and 3_47 are not observed. We thus conclude that the models with diffuse gas stripping reproduce the observed HI and NUV morphologies significantly better than the models without diffuse gas stripping.

\subsection{The Ho morphology}

The model $\mathrm{H} \alpha$ images consist of two components: (i) the HII regions ionized by young massive stars and (ii) diffuse ionized gas that is ionized by the stellar UV radiation or possibly by strong shocks induced by ram pressure stripping. The first component is modeled by the distribution of stellar particles with ages less than $20 \mathrm{Myr}$. This is the only component used for the images of the models without diffuse gas stripping. For the second component, the emission measure for all gas particles with temperatures lower than $10^{6} \mathrm{~K}$ was calculated: $E M=\rho^{2} d$, where $\rho$ is the gas density calculated with the SPH algorithm and the cloud diameter is given by $d=2\left(3 M_{\mathrm{cl}} /(4 \pi \rho)\right)^{1 / 3}$ with the cloud mass $M_{\mathrm{cl}}$. In the absence of a model for the ISM ionization fraction, we assume a constant ionization for the diffuse and dense gas. We then added the HII region component with a heuristic normalization, which worked best to insure that extraplanar HII regions are well visible within the emission of the diffuse ionized component. In these images, the surface brightness of the disk emission with respect to the extraplanar emission is realistic in a qualitative, but not quantitative, sense.

Fossati et al. (2018) observed an $\mathrm{H} \alpha$ tail whose high surface brightness part is clumpy and follows the NUV tail (upper-right panel of Fig. 1). A diffuse component of low surface brightness extends downwind to the south. In addition, low surface brightness filaments extend further from the tail to the south. Another low surface brightness $\mathrm{H} \alpha$ filament is detected close to the downturn in the northeast. As noted by Fossati et al. (2018), all filaments indicate the direction of the motion of the galaxy.

The resulting model $\mathrm{H} \alpha$ emission maps for the snapshots of the highest goodness of the diffuse gas stripping model are presented in Fig. A.1. All model snapshots show an $\mathrm{H} \alpha$ tail that has the same extent as the observed NUV tail. Most of the models show extraplanar linear filaments in the downwind region of the galactic disk. These filaments are thicker than the observed $\mathrm{H} \alpha$ filaments (see Sect. 3.3). They are labeled for model 4new_47 in the upper-left panel of Fig. A.1. Their lengths are comparable to those of the observed $\mathrm{H} \alpha$ filaments. All models show an extraplanar linear filament originating from the downturn region, which extends out of the disk toward the south. This filament has a north-south direction in models 4new_47, 1cnew_47, and 3dnew_47. It has a position angle of $\sim 135^{\circ}$ (i.e., it extends toward the southeast) in the other models. A second linear filament originating close to the galaxy center and extending into the downwind region is observed in models 4new_47, 1cnew_47, and 4anew_47. A third north-south filament is observed in the middle of the tail of model 4new_47. A fourth north-south filament starting from the outer edge of the $\mathrm{H} \alpha$ or NUV tail is present in models 4new_47, 1cnew_47, and 4anew_47. The direct comparison between model 4new_47 and the VESTIGE observations (Fig. 1) shows that this last filament might be interpreted as an extension of the gas tail. Because of the vertical orientation of its outermost part (compare to model 1cnew_47), we instead interpreted this feature as a filament.

Fossati et al. (2018) identified five extraplanar filaments in the downwind region: one originating close to the downturn region and four filaments in the tail region. Model 4new_47 with its four $\mathrm{H} \alpha$ filaments most closely resembles the observed $\mathrm{H} \alpha$ morphology. We therefore conclude that model 4new_47 best reproduces the deep VESTIGE $\mathrm{H} \alpha$ observations (lower-right panel of Fig. 1).

To demonstrate the effect of diffuse gas stripping on the model $\mathrm{H} \alpha$ emission distribution, we show the same model snapshots as in Fig. A.1 for the simulation without diffuse gas 

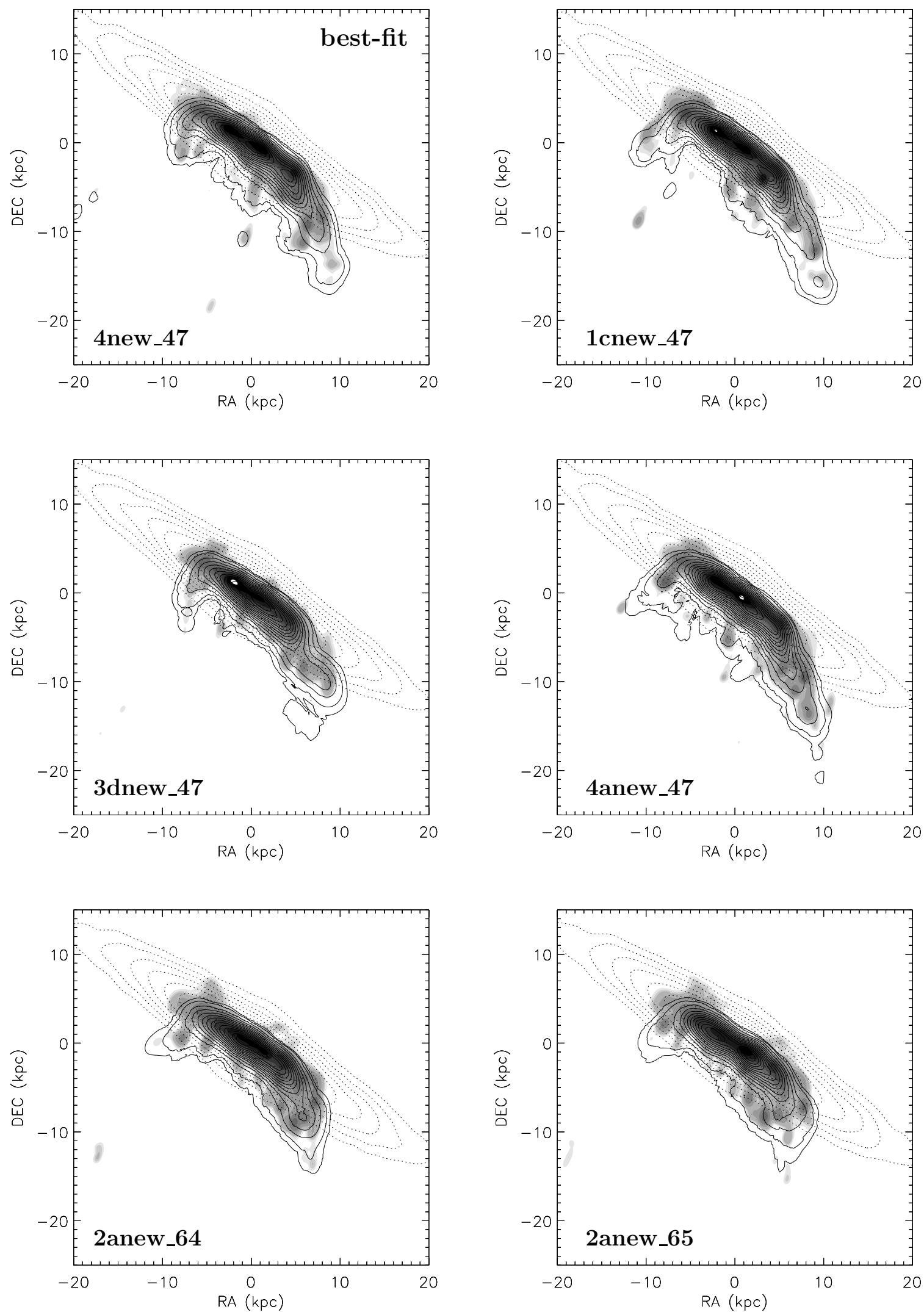

Fig. 4. Models with a diffuse ISM component. The grayscale represents NUV images, solid contours represent HI images, and dotted contours represent stellar images. For the spectral fitting, a variable nuisance parameter $\mathrm{Ly}_{\text {scale }}$ was used.

stripping in Fig. A.2. All model snapshots show an $\mathrm{H} \alpha$ tail comparable to that of the simulations with diffuse gas stripping. As expected, the number of extraplanar linear filaments is significantly decreased compared to the simulations with diffuse gas stripping. Only models 4_47, 1c_47, and 3d_47 show such a filament starting from the downturn region. However, its 
B. Vollmer et al.: A Virgo Environmental Survey Tracing Ionised Gas Emission (VESTIGE). VIII.
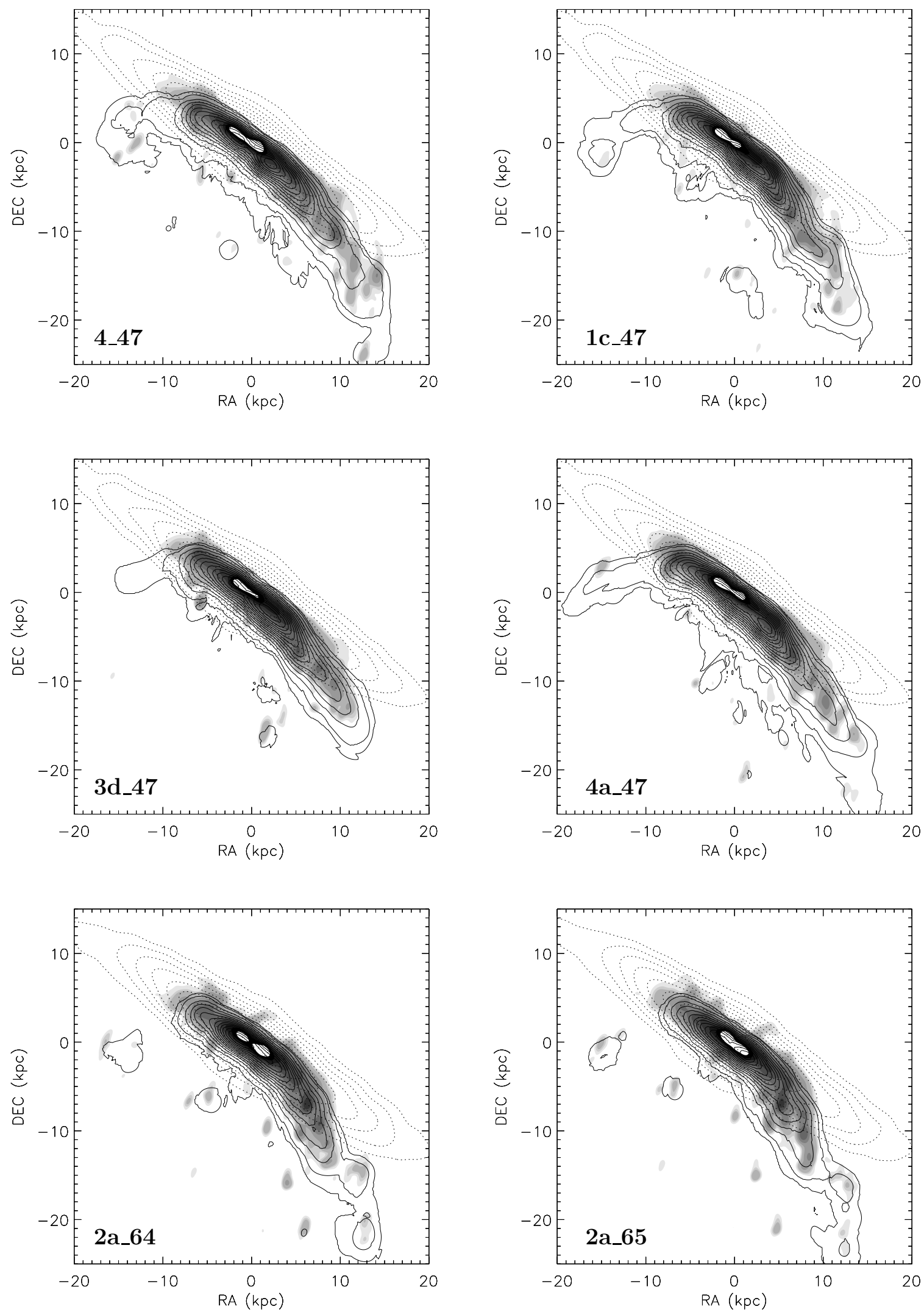

Fig. 5. Same as Fig. 4 but without a diffuse ISM component.

position angle differs significantly from zero (north-south direction). Vertical filaments along the projected direction of ram pressure stripping are only present in the diffuse gas because of its increased stripping efficiency. The ratio between tidal and

ram pressure acceleration is higher for the dense gas, which is thus less accelerated along the wind direction. Based on the absence of north-south filaments, we conclude that the presence of diffuse gas stripping increases the number of extraplanar $\mathrm{H} \alpha$ 

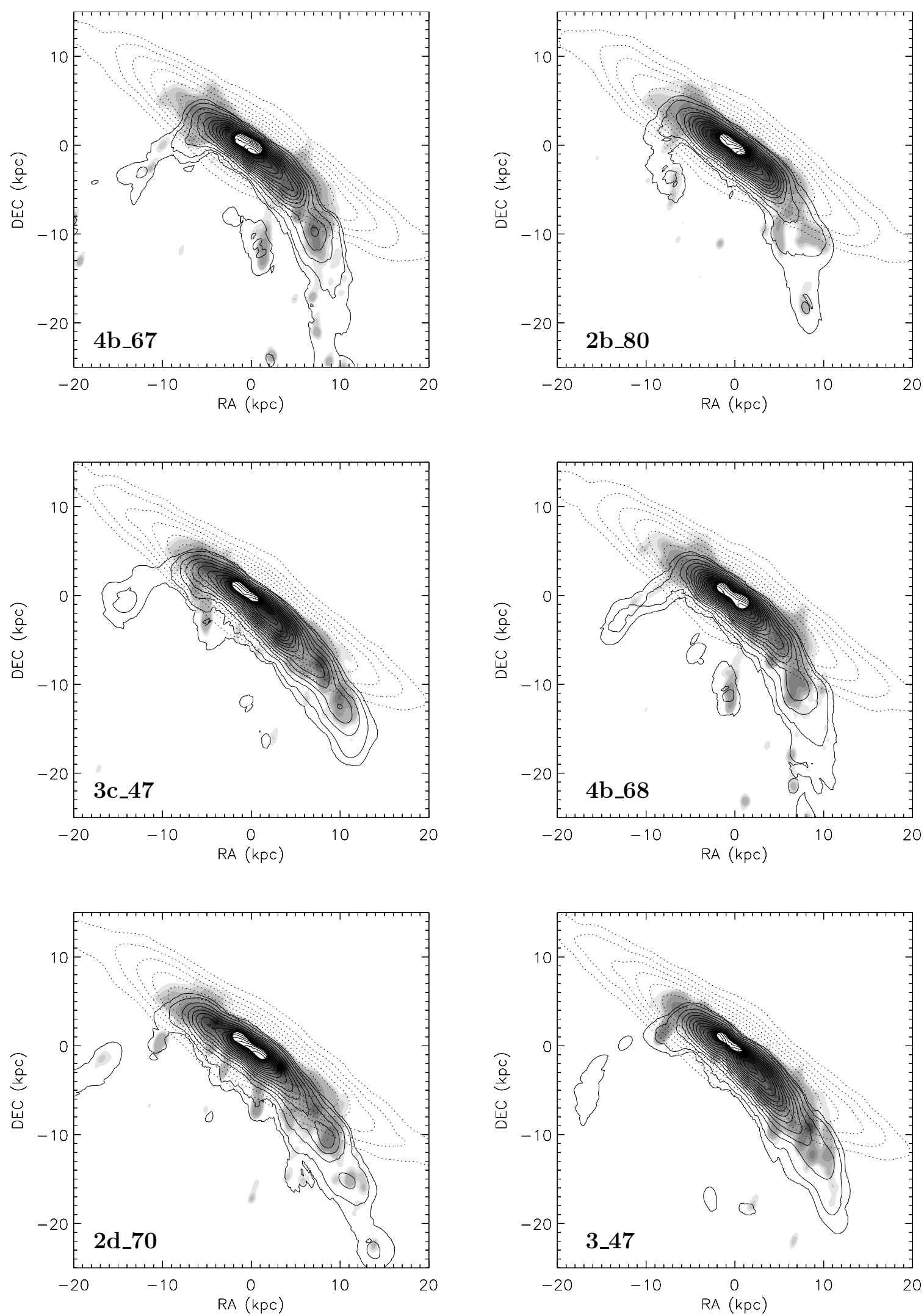

Fig. 6. NUV (grayscale), HI (solid contours), and stellar (dotted contours) images. Models without a diffuse ISM component are shown. For the spectral fitting, a variable nuisance parameter $\mathrm{Ly}_{\text {scale }}$ was used.

filaments in the downwind region and leads to a north-south alignment of these filaments. The simulations with diffuse gas stripping thus reproduce observations best.
The snapshots with the highest goodnesses for the models without diffuse gas are presented in Fig. A.3. The $\mathrm{H} \alpha$ tail is comparable to that of the previously discussed models. 
B. Vollmer et al.: A Virgo Environmental Survey Tracing Ionised Gas Emission (VESTIGE). VIII.

The extraplanar filaments starting from the downturn region are still present in all model snapshots. However, their position angle is not zero, contrary to the observations. Only model $2 d_{-} 70$ shows north-south filaments starting from the galactic disk and the tail region. Only the filament originating at the end of the tail deviates from the north-south alignment of the other filaments, in contrast to the corresponding filament 4 in model 4 new_47 (upper-left panel of Fig. A.1). We think that this difference is significant.

We conclude that the existence of north-south $\mathrm{H} \alpha$ filaments is a natural consequence of the presence of diffuse gas stripping. However, in rare cases, such filaments can also be created in models without diffuse gas stripping. Models 4new_47 and 2 d_70 reproduce the deep VESTIGE H $\alpha$ observations best.

\subsection{The best-fit model}

The results of the comparison between the model and observed HI, NUV, and deep $\mathrm{H} \alpha$ emission distributions are presented in Table 3. Model 4new_47 reproduces the HI and $\mathrm{H} \alpha$ emission distribution in the downturn and tail region in a satisfactory way. Moreover, the overall morphology of the observed NUV emission is qualitatively reproduced by the model. However, the NUV disk in the tail region is less extended than observed. This is mainly an effect of NUV surface brightness, which is lower in the model NUV tail compared to observations (see also the UV photometry in Figs. A.4-A.6). As a result, the offset between the $\mathrm{H} \alpha$ and NUV tails is much less pronounced relative to the observations. Moreover, the model NUV emission of the downturn region shows too much extraplanar emission on the downwind side compared to the GALEX observations. The observed offset between the NUV and the HI tail is absent in this model. This offset is only present in model 1cnew_47, and the observed NUV emission distribution is reproduced best by this model snapshot. However, model 1cnew_47 shows too much extraplanar HI emission in the downturn region. Based on the comparison of all observed characteristics with our model snapshots (Table 3), we conclude that model 4new_47 fits the available observations, including the optical spectra, best.

\subsection{The optical spectra}

The model optical spectra and the model SED for the bestfit model 4new_47 (including diffuse gas stripping and a variable nuisance parameter $\mathrm{Ly}_{\text {scale }}$ ) are presented in Figs. A.4-A.9 together with the SFH for the regions labeled in Fig. 3. The optical-to-NIR SED depends only on the SFH prior to the onset of the simulations and is always well reproduced by the models. The FUV and NUV flux densities are well reproduced by the model in regions 2,3 , and 11 . They are somewhat underestimated by the model in downturn regions 12 and 13 and overestimated in the outer tail region 1. The optical spectra of all positions are well reproduced by the model. For a seamless comparison, we added the results from Fossati et al. (2018) for each position to Figs. A.4-A.9 ${ }^{3}$. It becomes clear that the good resemblance between the model and observations for all regions is thanks to the good resemblance between the observed and modeled SFHs. Whereas the SFHs of Fossati et al. (2018) are analytical with exponentially declining SFR after gas stripping, the model SFHs show more variations. If the model SFHs show a late peak of the SFR after the general decline of the SFR

\footnotetext{
3 The spectra of regions $1,3,11,12$, and 13 are not shown in Fossati et al. (2018).
}

Table 5. Nuisance parameter $\mathrm{Ly}_{\text {scale }}$.

\begin{tabular}{lcccccc}
\hline \hline Region (Fig. 3) & R1 & R2 & R3 & R11 & R12 & R13 \\
\hline Fossati et al. & 0.34 & 0.35 & 0.75 & 0.60 & 0.50 & 0.49 \\
4new_47 & 1.17 & 1.21 & 1.83 & 1.60 & 0.90 & 1.42 \\
4anew_60 & 0.73 & 1.20 & 1.39 & 0.57 & 0.84 & 0.91 \\
4b_67 & 1.39 & 1.13 & 1.39 & 1.28 & 1.09 & 0.89 \\
2b_80 & 0.64 & 0.34 & 1.21 & 0.62 & 0.84 & 0.97 \\
\hline
\end{tabular}

(regions 2 and 12), the onset of the decline occurs earlier than that derived with a simple analytical function, as in Fossati et al. (2018), because this additional young stellar population has to be compensated for via a removal of the stellar populations that were created just prior to the onset of SFR quenching. We conclude that our best-fit model 4new_47 reproduces the observed optical spectra and SED in a satisfactory way.

\subsection{A constant nuisance parameter $L y_{\text {scale }}$}

For the calculation of the model goodness, the nuisance parameter Ly $y_{\text {scale }}$ was varied systematically between $1 / 3$ and 3 to search for the value that led to the highest goodness. The parameter $\mathrm{Ly}_{\text {scale }}$ takes into account that either some UV flux from the massive stars located in a given aperture escapes the region without ionizing the gas or that massive star outside the aperture ionize gas located inside the aperture. In addition, ram pressure induced shock can in principle lead to the ionization of the ambient gas. In the first case, $\mathrm{Ly}_{\text {scale }}$ is less than one, while in the last two cases it exceeds unity. The resulting $\mathrm{Ly}_{\text {scale }}$ parameters for the Fossati et al. (2018) model SFH along with the SFHs derived from the dynamical models with the highest goodnesses are presented in Table 5.

The analytical SFHs of Fossati et al. (2018) lead to $\mathrm{Ly}_{\text {scale }}$ values of less than one for all regions. Its values range from 0.33 to 0.75 . The $\mathrm{Ly}_{\text {scale }}$ parameter found for the dynamical models can exceed unity, ranging from 0.33 to 1.83 . Our best-fit model in particular, 4new_47, leads to $\mathrm{Ly}_{\text {scale }}>1$ in five out of six regions. Since these values are not exceedingly high, we think that this model is very much acceptable. Values exceeding unity could imply that ram-pressure induced shock probably plays a role in ionizing the ambient ISM. However, the high flexibility of the fitting model and the point spread function mismatch between the spectrum and the photometry prevent such a conclusion.

All spectra and SEDs were recalculated using the values of the $\mathrm{Ly}_{\text {scale }}$ parameter found by Fossati et al. (2018). The resulting model snapshots with the highest goodnesses are presented in Table 3 for the simulations with and without a diffuse gas stripping. As stated previously, all goodnesses of the models without diffuse gas stripping are smaller than those of the model with diffuse gas stripping. In addition, a fixed $\mathrm{Ly}_{\text {scale }}$ parameter for each region leads to the highest goodnesses for snapshots at later times compared to models with a variable $\mathrm{Ly}_{\text {scale }}$ parameter.

The resulting model HI, NUV, and $\mathrm{H} \alpha$ images are presented in Figs. D.1-D.4. Whereas the model HI distributions are similar to the models with a variable $\mathrm{Ly}_{\text {scale }}$ parameter, the snapshots from the models that include a fixed $\mathrm{Ly}_{\text {scale }}$ parameter all show extraplanar NUV emission in the upturn region on the upwind side (Figs. D.1 and D.3). This NUV emission, which is not observed, is due to massive stars created in the tail region that had the time to rotate into the upturn region (see Sect. 5.1). In 
addition, none of the models with a fixed $\mathrm{Ly}_{\text {scale }}$ parameter show the vertical low surface brightness $\mathrm{H} \alpha$ filaments on the downwind side of the NUV or $\mathrm{H} \alpha$ tail. We therefore conclude that the models with a fixed $\mathrm{Ly}_{\text {scale }}$ parameter should be discarded and that this parameter primarily plays a nuisance role in the fit rather than having a physical meaning.

\section{Discussion}

The inclusion of diffuse gas stripping significantly changed the results of our simulations. Since a variable $\mathrm{Ly}_{\text {scale }}$ parameter leads to the highest goodnesses, we do not consider the models with a constant $\mathrm{Ly}_{\text {scale }}$ parameter in the following analysis. When the tail region is considered separately, the timesteps of interest are $\sim 50$ Myr before peak ram pressure when diffuse gas stripping is included in the simulations (Table 4).

This is expected because the gas that is pushed into the tail becomes diffuse and is stripped with a higher efficiency than the denser gas. Unexpectedly, the opposite trend is found for the downturn region: The timesteps of interest are $\sim 70 \mathrm{Myr}$ before peak ram pressure when diffuse gas stripping is not included. The reason for this behavior is the following: As expected, the diffuse gas is stripped rapidly out of the galactic disk on the windward side when diffuse gas stripping is included. At the same time, the gas that remains within the disk is compressed to higher densities compared to the simulations without diffuse gas stripping. This results in a more compact gas distribution at $t \gtrsim 200$ Myr after the onset of the simulations, that is to say, the surface density contrast of the gas located within the galactic disk is enhanced. Since more time is needed to push the outer dense arms to smaller galactic radii, the timesteps of interest for the downturn (windward) side are delayed when diffuse gas stripping is included. The bulk of the star formation occurs in the dense gas arms.

The situation changes when both, the downturn and tail regions, are fitted together (Table 3). Generally, the $\mathrm{H} \alpha$ and NUV emission distributions of the downturn region are more often reproduced when diffuse gas stripping is not included in the simulation. Overall, the HI distribution of the downturn region is hard to reproduce (4new_47 and 4_d70). The best reproductions of the observed HI distribution of the tail are only found in models with diffuse gas stripping. The $\mathrm{H} \alpha$ emission distribution of the tail region is often reproduced when diffuse gas stripping is not included, but only for peak and post-peak models. Most frequently, post-peak models are not allowed, because they show an NUV horn feature on the leeward side of the downturn region (see Sect. 5.1).

While the two acceptable models with diffuse gas stripping according to the total goodness (4new_47 and 1cnew_47) have $\Delta t_{\text {peak }}=t-t_{\text {peak }} \sim-170 \mathrm{Myr}$ (i.e., they are pre-peak models), the two acceptable models without diffuse gas stripping (4b_67 and $\left.2 \mathrm{~b} \_80\right)$ are post-peak models $\left(\Delta t_{\text {peak }}>0 \mathrm{Myr}\right)$. Based on the comparison between the model and observed HI, NUV, and $\mathrm{H} \alpha$ distributions, the pre-peak models 4new_47 and 1cnew_47 are clearly preferred (Sect. 5.3).

\subsection{The best-fit model}

The best-fit model is 4 new 47 , with a maximum ram pressure of $p_{\max }=6000 \mathrm{~cm}^{-3}\left(\mathrm{~km} \mathrm{~s}^{-1}\right)^{2}$, a width of the Lorentzian temporal profile of $t_{\mathrm{HW}}=200 \mathrm{Myr}$, and a time to peak ram pressure of $\Delta t=t-t_{\text {peak }}=-140 \mathrm{Myr}$. The face-on projection of the distribution of the total gas surface density at the beginning of the simulation and at $\Delta t=-140 \mathrm{Myr}$ is presented in Fig. 7. The ini-
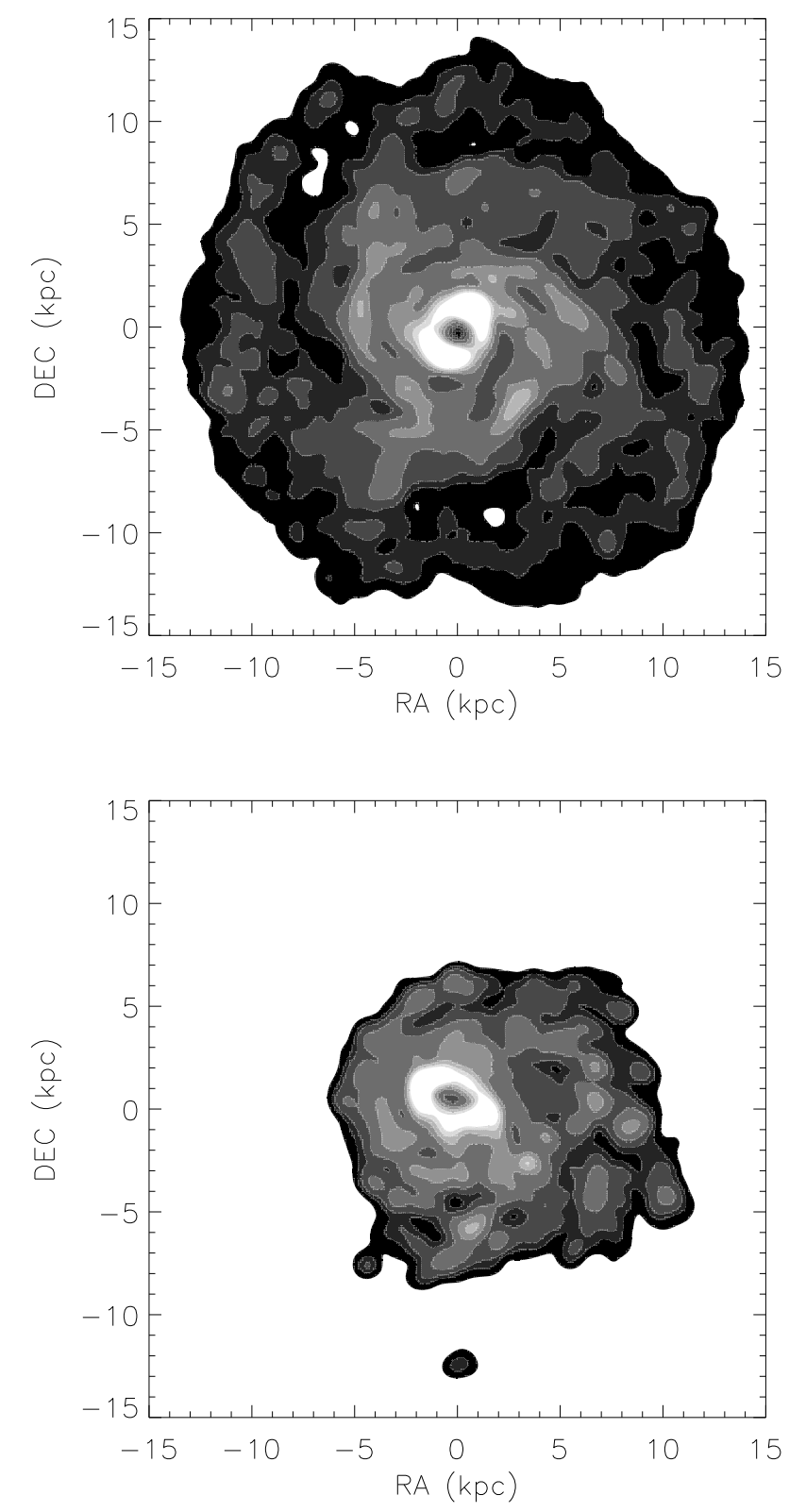

Fig. 7. Face-on projection of the distribution of the total gas surface density at the beginning of the simulation (upper panel) and at $\Delta t=$ $-140 \mathrm{Myr}$ (lower panel). The contours correspond to 1, 3, 5, 10, 20, 30, 40 , and $50 M_{\odot} \mathrm{pc}^{-2}$. The resolution is $820 \mathrm{pc}$.

tial gas distribution of the galaxy has multiple spiral arms with surface densities between 10 and $20 M_{\odot} \mathrm{pc}^{-2}$. During the ram pressure stripping event, we observe a highly nonlinear interaction between these arms and the induced gas compression. The gas distribution at $\Delta t=-140 \mathrm{Myr}$ has a compressed windward side and a gas tail with a multi-arm structure on the opposite side.

The model includes the stripping of diffuse gas and a variable $\mathrm{Ly}_{\text {scale }}$ parameter for the calculation of the model optical spectra and thus the model goodness. The interaction parameters are close to those found by Vollmer et al. $(2012 \mathrm{a}, \mathrm{b}): p_{\max }=$ $5000 \mathrm{~cm}^{-3}\left(\mathrm{~km} \mathrm{~s}^{-1}\right)^{2}, t_{\mathrm{HW}}=100 \mathrm{Myr}$, and $\Delta t=-100 \mathrm{Myr}$. The Vollmer et al. (2012a,b) model was not able to reproduce the UV color in the stripped gas-free regions because the width of the Lorentzian profile was too small. A larger width permits an earlier gas stripping, which is consistent with the available UV 
observations and the optical spectra. In addition, the inclusion of diffuse gas stripping is essential. Only when this ingredient is added to the simulations does the gas leave the galactic disk early enough to lead to FUV and NUV flux densities and optical spectra consistent with observations. The diffuse gas stripping leads to the formation of linear filaments of the diffuse ionized gas that is present in deep VESTIGE H $\alpha$ observations. The model filaments are thicker than the observed filaments because of the coarse spatial resolution of the diffuse gas particles. It should be noted that pronounced filamentary structures are naturally produced in simulations whenever magnetic fields are taken into account (Ruszkowski et al. 2014; Tonnesen \& Stone 2014). To determine whether or not this effect is ubiquitous, it has to be shown for other galaxies undergoing ram pressure stripping.

\subsection{Ram pressure stripping of cosmic ray electrons}

NGC 4330 does not show an asymmetric ridge of polarized emission, in contrast to other ram-pressure stripped galaxies (Vollmer et al. 2012a,b). This is due to the NGC 4330 projection that places the region of compressed gas along the line-ofsight. On the other hand, Vollmer et al. $(2012 \mathrm{a}, \mathrm{b})$ discovered a tail structure bending southward in their $6 \mathrm{~cm}$ radio continuum data (upper panel of Fig. 8). This tail structure is also visible in the VIVA $20 \mathrm{~cm}$ data (lower panel of Fig. 8). In addition, an outer tail of low surface brightness extending toward the southwest is detected at $20 \mathrm{~cm}$.

The tail, which is detected at 6 and $20 \mathrm{~cm}$, bends to the south at the southwestern edge of the star-forming disk. It coincides with the northern part of the low surface brightness diffuse $\mathrm{H} \alpha$ emission. The cosmic ray electrons produced in the luminous HII regions of the galactic disk are most probably pushed to the south by ram pressure. This hypothesis is corroborated by the vertical magnetic field lines detected within the radio continuum tail (Vollmer et al. 2013). The spatial coincidence of the radio continuum and diffuse $\mathrm{H} \alpha$ tails suggests that both gas phases are stripped together.

We do not observe a steepening of the spectral index in the 6 and $20 \mathrm{~cm}$ tail, as expected for an aging cosmic electron population. The spectral index is given by $\alpha=\ln \left(f_{6} / f_{20}\right) / \ln \left(v_{6} / v_{20}\right)$, where $f$ is the surface brightness or flux density. For a purely aging population of cosmic ray electrons, the evolution of the flux density is given by $f=f_{0} \times \exp \left(-t / t_{\text {syn }}\right)$. The time that is needed for a steepening of the spectral index from an initial value $\alpha_{\text {in }}$ to a final value $\alpha_{\text {end }}$ can be calculated in the following way:

$t_{\mathrm{SI}}=\frac{\left(\alpha_{\text {in }}-\alpha_{\text {end }}\right) \ln \left(v_{6} / v_{20}\right)}{1 / t_{\mathrm{syn}, 6 \mathrm{~cm}}-1 / t_{\mathrm{syn}, 20 \mathrm{~cm}}}$.

Based on equipartition between the energy densities of the cosmic ray electrons and the magnetic field, we estimate the magnetic field strength to be $B \sim 6-7 \mu \mathrm{G}$ in the tail region, assuming an extent along the line-of-sight of $l \sim 4 \mathrm{kpc}$. The associated synchrotron timescale at $6 \mathrm{~cm}$ is then

$t_{\text {syn }}=4.5 \times 10^{7}\left(\frac{B}{10 \mu \mathrm{G}}\right)^{-3 / 2} v_{\mathrm{GHz}}^{-1 / 2} \mathrm{yr} \sim 40 \mathrm{Myr}$.

If we assume that $\alpha_{\text {in }}-\alpha_{\text {end }} \lesssim 0.2$ in the 6 and $20 \mathrm{~cm}$ tail, the timescale for the steepening of the spectral index is $t_{\mathrm{SI}} \lesssim 24 \mathrm{Myr}$. With a north-south extent of $3.5 \mathrm{kpc}$, the southward bulk velocity is $\gtrsim 140 \mathrm{~km} \mathrm{~s}^{-1}$ within the sky plane.

Assuming that the outer radio continuum tail is barely detected at a $2 \sigma$ level at $6 \mathrm{~cm}$, we estimate a spectral index
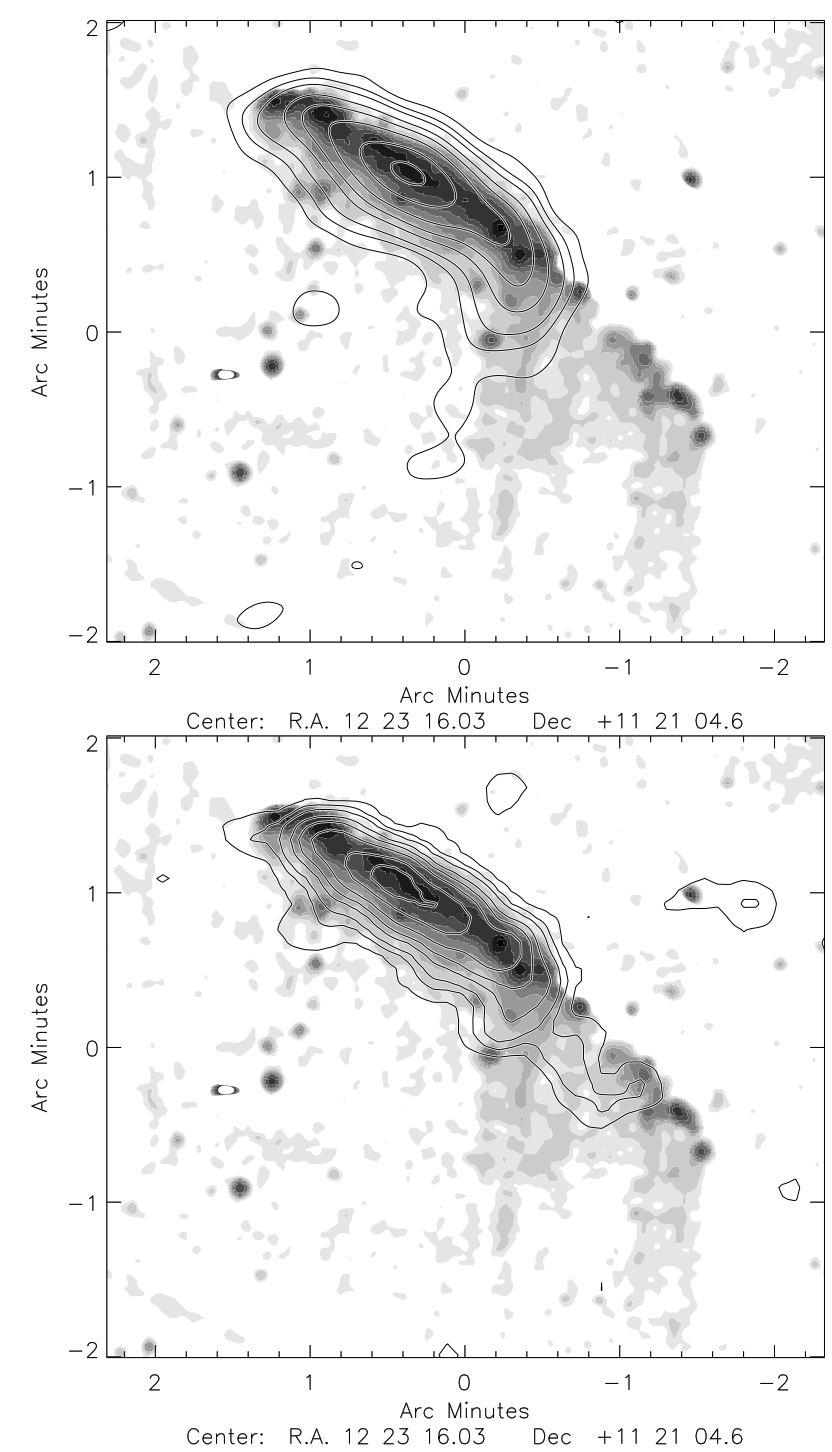

Fig. 8. Radio continuum emission (contours) on the VESTIGE $\mathrm{H} \alpha$ map. Upper panel: emission at $6 \mathrm{~cm}$. The contour levels are $(1,2,3,5,7,10,15,20,30,50,100) \times 50 \mu \mathrm{Jy}_{\text {beam }}^{-1}$. The resolution is $\left(22^{\prime \prime} \times 22^{\prime \prime}\right)$. Lower panel: emission at 20cm from VIVA (Chung et al. 2009). The contour levels are $(1,2,3,5,7,10,15,20,30,50,100) \times$ $75 \mu \mathrm{Jy}$ beam $^{-1}$. The resolution is $\left(19.4^{\prime \prime} \times 15.4^{\prime \prime}\right)$.

between 6 and $20 \mathrm{~cm}$ of $\sim-1.5$. With an initial spectral index of $\alpha_{\text {in }}=-0.7$ to -0.5 , a final spectral index $\alpha_{\text {in }}=-1.5$, and a magnetic field strength of $B=6 \mu \mathrm{G}$, we obtain $t_{\mathrm{SI}} \sim 90$ 120 Myr. If the cosmic ray electrons were produced in the normally star-forming disk before gas stripping, $t_{\mathrm{SI}}$ should be comparable to the star formation quenching time derived for that region, which corresponds to region 2 in Fossati et al. (2018). Whereas Fossati et al. (2018) determined a quenching time of 175 Myr based on their simple analytical SFHs, our preferred model shows that a more recent star formation episode (about 50 Myr ago; Fig. A.5) is also consistent with the data. It is not clear if such an episode can create enough cosmic ray electrons to make the synchrotron emission detectable. On the other hand, a somewhat lower magnetic field strength of $B=4-5 \mu \mathrm{G}$ would lead to $t_{\mathrm{SI}} \sim 175 \mathrm{Myr}$. We thus conclude that a scenario in which the cosmic ray electrons were produced in the healthy disk before gas stripping, and were then pushed southward together with the diffuse ionized gas, is consistent with the available data. 
It is surprising that there is no $20 \mathrm{~cm}$ emission associated with the upper part of the outer $\mathrm{H} \alpha$ tail where we see compact emission, which is most probably produced by HII regions. Either these HII regions are too young and have not yet produced cosmic ray electrons, or the production of cosmic ray electrons by these regions is low. We suggest that the present star formation in the outer tail is sporadic and low level and that this explains the absence of a significant amount of cosmic ray electrons there.

\subsection{How to ionize the stripped diffuse gas}

It is surprising that the surface brightness of the $\mathrm{H} \alpha$ filaments does not systematically decrease southward. These filaments therefore need to be confined by the ambient ICM for $\sim 60 \mathrm{Myr}$ without recombining, that is to say, there must be a continuous ionization mechanism. In the following, we argue that the stripped diffuse gas is ionized by thermal conduction. With a recombination timescale $t_{\mathrm{rec}}=0.1\left(n_{\mathrm{e}} / 1 \mathrm{~cm}^{-3}\right)^{-1} \mathrm{Myr}=60 \mathrm{Myr}$, this yields an electron density of $n_{\mathrm{e}} \sim 2 \times 10^{-3} \mathrm{~cm}^{-3}$. The mean electron density of the $\mathrm{H} \alpha$ filaments can be estimated by the relation

$L(\mathrm{H} \alpha)=n_{\mathrm{e}} n_{\mathrm{p}} \alpha_{\mathrm{H} \alpha}^{\mathrm{eff}} V \phi_{\mathrm{V}} h v_{\mathrm{H} \alpha}$

(Osterbrock \& Ferland 2006), where $n_{\mathrm{p}}=n_{\mathrm{e}}$ is the proton density, $\alpha_{\mathrm{H} \alpha}^{\text {eff }}=1.17 \times 10^{-13} \mathrm{~cm}^{3} \mathrm{~s}^{-1}$ is the $\mathrm{H} \alpha$ effective recombination coefficient, $V$ is the volume of the emitting region, $\phi_{\mathrm{V}}$ is its volume filling factor, $h$ is the Planck constant, and $v_{\mathrm{H} \alpha}$ is the frequency of the $\mathrm{H} \alpha$ transition. The volume $V$ is the product of the emitting surface and the extent along the line-of-sight $l \sim 4 \mathrm{kpc}$. Following Boselli et al. (2016), we assume $\phi_{\mathrm{V}}=0.1$ and $[\mathrm{NII}] / \mathrm{H} \alpha=0.5$. With a mean $\mathrm{H} \alpha+[\mathrm{NII}]$ surface brightness of $4 \times 10^{-18} \mathrm{erg} \mathrm{cm}^{-2} \mathrm{~s}^{-1} \operatorname{arcsec}^{-2}$ (Fossati et al. 2018), we obtain a mean electron density of $n_{\mathrm{e}}=0.05 \mathrm{~cm}^{-3}$. With an emitting surface of $25 \mathrm{kpc}^{2}$, the mass of ionized gas in the filaments is about $10^{7} M_{\odot}$.

Since the derived mean electron density of the filaments exceeds that derived from the recombination timescale, the hydrogen atoms in the filaments have to be continuously ionized. Boselli et al. (2016) reached the same conclusion for the long, low surface brightness $\mathrm{H} \alpha$ tails of NGC 4569, another ram-pressure stripped galaxy in the Virgo cluster. The source of ionization might be (i) collisional ionization through shocks, (ii) collisional ionization through the thermal electrons of the ambient ICM that confines the filaments, or (iii) radiative ionization by embedded massive stars. We discard the third hypothesis because the gas density in the filaments is not high enough to permit continuous star formation. Hypothesis (i) is improbable because the direction of filaments is aligned with the ram pressure wind and it is difficult to perceive how ram-pressure induced shocks can pervade the entirety of the filaments.

We are thus left with hypothesis (ii): If we assume that the timescale for evaporation by the ICM equals the collisional ionization timescale by ICM-ISM collisions $t_{\mathrm{ICM}-\mathrm{ISM}}=t_{\mathrm{evap}}=$ $10\left(N_{\mathrm{H}} / 10^{20} \mathrm{~cm}^{-2}\right) \mathrm{Myr}$ (Vollmer et al. 2001), and if the recombination timescale is $t_{\mathrm{rec}}=0.1\left(0.3 \mathrm{~cm}^{-3} / 1 \mathrm{~cm}^{-3}\right)^{-1} \mathrm{Myr}=0.3 \mathrm{Myr}$, we obtain a hydrogen column density of $N_{\mathrm{H}} \sim 3 \times 10^{18} \mathrm{~cm}^{-2}$. This is well below the VIVA detection limit $\left(\sim 2 \times 10^{19} \mathrm{~cm}^{-2}\right.$; Abramson et al. 2011). We thus suggest that the most probable mechanism for the ionization of the $\mathrm{H} \alpha$ filaments is collisions between the ICM thermal electrons and the hydrogen atoms of the filaments (as in ionized gas filaments in cooling flow clusters; see e.g., McDonald et al. 2010).

\section{Summary and conclusions}

The edge-on Virgo spiral galaxy NGC 4330 shows signs of ongoing ram pressure stripping: a truncated HI disk together with a one-sided tail (Chung et al. 2007, 2009), a UV tail associated with the HI tail (Abramson et al. 2011), a truncated $\mathrm{H} \alpha$ disk (Abramson et al. 2011), and an $\mathrm{H} \alpha$ tail with vertical low surface density filaments (Fossati et al. 2018; upper panels of Fig. 1). There is no asymmetric ridge of polarized radio continuum emission as observed in other ram-pressure stripped Virgo galaxies because the compression is occurring in the sky plane (Vollmer et al. 2012a,b). A previous dynamical model could reproduce all observed characteristics except the UV color of the stripped gas-free regions of the outer galactic disk (Vollmer et al. 2012a,b). Since the UV color depends on the SFH of the observed region, the stripping timescale of the dynamical model was not consistent with observations.

Fossati et al. (2018) obtained VLT FORS2 deep optical spectra along the major axis of NGC 4330. In addition, they collected literature photometry in 15 bands from the FUV to the FIR. Star formation histories in apertures along the major axis of the galaxy were constructed by means of stellar population fitting to the joint observational data: the SED and the optical spectrum. Based on an analytical model of quenched SFHs, they found that the outermost radii of the galactic disk were stripped $\sim 500 \mathrm{Myr}$ ago, while the stripping reached the inner $5 \mathrm{kpc}$ in the last $100 \mathrm{Myr}$.

We present new dynamical simulations to reproduce all observed characteristics of NGC 4330: the HI, UV, and H $\alpha$ morphologies together with the SEDs and the optical spectra. The dense gas is modeled by a sticky particle scheme (Sect. 3). The influence of galactic structure (i.e., spiral arms) on the gas stripping process was studied by delaying the ram pressure peak with respect to the beginning of the simulations. Most importantly, we introduce a new recipe for the stripping of diffuse gas with a low density and a high volume filling factor (Sect. 3.3):

Warm diffuse gas: Once a gas particle has left the galactic disk and its density falls below a critical density $n_{\text {crit }}^{\text {warm }}$, ram pressure is increased by $\left(0.044 \mathrm{~cm}^{-3} / n_{\mathrm{ISM}}\right)^{2 / 3}$.

Hot $\left(T>10^{6} \mathrm{~K}\right)$ diffuse gas: temperatures are assigned to the gas particles. Once a gas particle has left the galactic disk and its density falls below a critical density, $n_{\text {crit }}^{\text {hot }}$ ICM-ISM mixing sets in and immediately increases the temperature to $9 \times 10^{6} \mathrm{~K}$. If the density of the mixed gas increases through compression, it can cool through X-ray emission according to its density and temperature. The acceleration due to ram pressure is increased by a factor of ten once the temperature of a particle exceeds $T=10^{5} \mathrm{~K}$. Moreover, the equation of motion for the hot gas particles was modified by adding the acceleration caused by the gas pressure gradient. We do not solve an explicit energy equation, and the calculation of hydrodynamic effects is approximate.

We ran 50 simulations with five different Lorentzian temporal ram pressure profiles, with five different delays between the simulation onset and peak ram pressure, and with (newprof) and without (prof) diffuse gas stripping (Table 2). Model photometry and spectra were calculated for every timestep $(\Delta t=10 \mathrm{Myr})$ of the 50 simulations. In addition, we decided to handle the Ly scale nuisance parameter (Sect. 4) in two different ways: (i) We set it to the fixed values found by Fossati et al. (2018), and (ii) we treated it as a free parameter and kept the value that led to the best fit to observations. We then searched for the 12 timesteps with the highest goodnesses for the downturn, tail, and both (downturn plus tail) regions (Table 3 ). The individual regions (downturn and tail) were generally not best fitted by the same model nor by 
the same timestep. This is due to the limitations of our model, where the gas stripping is treated with relatively simple recipes (see Sect. 3.3), and the limited number of temporal ram pressure profiles. Our analysis is based on the combined goodness of both regions.

Based on the direct comparison between our model snapshots and the available observations (Table 3), we conclude that the inclusion of diffuse gas stripping:

1. Significantly changes the gas, NUV (Figs. 4 and 5), and $\mathrm{H} \alpha$ morphologies (Figs. A.1 and A.2) of the simulations.

2. Naturally leads to vertical low surface density filaments in the downwind region of the galactic disk (Fig. A.1). These filaments occur less frequently in the simulations without diffuse gas stripping (Figs. A.2 and A.3).

3. Leads to better joint fits to the SEDs and optical spectra (Table 3). In general, the SEDs and optical spectra of the downturn and tails regions fitted separately are best reproduced by different model snapshots (Table 3). We based our selection of the best-fit model on the goodness of the joint fit of the downturn and tail regions.

4. Significantly improves the resemblance between the model and observations.

The HI, NUV, and $\mathrm{H} \alpha$ morphologies of the model snapshots that best reproduce the SEDs and optical spectra are sufficiently different to permit a selection of best-fit models. Model 4new_47 is our preferred model (Table 3, lower panels of Fig. 1), with a maximum ram pressure of $p_{\max }=6000 \mathrm{~cm}^{-3}\left(\mathrm{~km} \mathrm{~s}^{-1}\right)^{2}$, a width of the Lorentzian temporal profile of $t_{\mathrm{HW}}=200 \mathrm{Myr}$, and a time to peak ram pressure of $\Delta t=-140 \mathrm{Myr}$. The model includes the stripping of diffuse gas and a variable $\mathrm{Ly}_{\text {scale }}$ parameter for the calculation of the model optical spectra. A variable $\mathrm{Ly}_{\text {scale }}$ nuisance parameter leads not only to higher goodnesses (i.e., better joint fits to the SEDs and optical spectra), as expected, but also to a much better reproduction of the observed $\mathrm{H} \alpha$ and NUV images. The interaction parameters are close to those found by Vollmer et al. (2012a,b), who found a time to peak ram pressure of $\sim 100 \mathrm{Myr}$.

The radio continuum morphology of the $\mathrm{H} \alpha$ tail is twofold: (i) a tail of relatively high surface brightness detected at $6 \mathrm{~cm}$ and $20 \mathrm{~cm}$ and originating from the southwestern part of the galactic disk, which still actively forms stars; and (ii) a low surface brightness tail associated with the outer $\mathrm{H} \alpha$ tail, with a steep spectral index only detected at $20 \mathrm{~cm}$ (Fig. 8). The magnetic field strength of both radio continuum tails is estimated to be $B \sim 6-7 \mu \mathrm{G}$. The spatial coincidence of the radio continuum and diffuse $\mathrm{H} \alpha$ tail suggests that both gas phases are stripped together. The southward bulk motion of the $6 \mathrm{~cm}$ and $20 \mathrm{~cm}$ is about $150 \mathrm{~km} \mathrm{~s}^{-1}$, with a timescale of $\sim 25 \mathrm{Myr}$. For the outer part of the $\mathrm{H} \alpha$ tail, a scenario in which the cosmic ray electrons were produced in the healthy disk before gas stripping and were then pushed southward together with the diffuse ionized gas is consistent with the available data. We suggest that the star formation in the outer tail is sporadic and low level and that this explains the absence of a significant amount of cosmic ray electrons there.

Since the surface brightness of the $\mathrm{H} \alpha$ filaments does not systematically decrease southward (upper-right panel of Fig. 1), they need to be confined by the ambient ICM for $\sim 60 \mathrm{Myr}$ without recombining. We suggest that the mixed ISM is ionized via collisions with the thermal electrons of the ambient ICM that confines the filaments.
The modeling of diffuse gas stripping represents an important step toward understanding the reaction of the multiphase ISM to ram pressure. The inclusion of diffuse gas stripping improves the resemblance between the model and observations (SEDs and optical spectra) and naturally gives rise to extended low surface density filaments oriented along the wind direction (diffuse $\mathrm{H} \alpha$ emission). More detailed simulations of cluster galaxies affected by ram pressure are needed to confirm our findings.

Acknowledgements. BV would like to thank R. Beck for useful discussions. We are grateful to the CFHT team who assisted us in the observations: T. Burdullis, D. Devost, B. Mahoney, N. Manset, A. Petric, S. Prunet, K. Withington. The VESTIGE project is financially supported by the "Programme National de Cosmologie and Galaxies" (PNCG). M. F. has received funding from the European Research Council (ERC) under the European Union's Horizon 2020 research and innovation programme (Grant agreement No. 757535). We would like to thank the anonymous referee for her/his comments which helped to improve the article.

\section{References}

Abramson, A., Kenney, J. D. P., Crowl, H. H., et al. 2011, AJ, 141, 164 Appenzeller, I., Fricke, K., Fürtig, W., et al. 1998, Messenger, 94, 1 Boissier, S., \& Prantzos, N. 2000, MNRAS, 312, 398

Boselli, A., Boissier, S., Cortese, L., et al. 2006, ApJ, 651, 811

Boselli, A., Cuillandre, J. C., Fossati, M., et al. 2016, A\&A, 587, A68

Boselli, A., Fossati, M., Ferrarese, L., et al. 2018, A\&A, 614, A56

Bruzual, G., \& Charlot, S. 2003, MNRAS, 344, 1000

Buchner, J., Georgakakis, A., Nandra, K., et al. 2014, A\&A, 564, A125 Calzetti, D., Armus, L., Bohlin, R. C., et al. 2000, ApJ, 533, 682

Chung, A., van Gorkom, J. H., Kenney, J. D. P., et al. 2007, ApJ, 659, L115 Chung, A., van Gorkom, J. H., Kenney, J. D. P., et al. 2009, ApJ, 138, 1741 Crowl, H. H., \& Kenney, J. D. P. 2008, AJ, 136, 1623

Feroz, F., \& Hobson, M. P. 2008, MNRAS, 384, 449

Feroz, F., \& Skilling, J. 2013, Am. Inst. Phys. Conf. Ser., 106,

Feroz, F., Hobson, M. P., \& Bridges, M. 2009, MNRAS, 398, 1601

Fossati, M., Mendel, J. T., Boselli, A., et al. 2018, A\&A, 614, A57

Gunn, J. E., \& Gott, J. R. 1972, ApJ, 176, 1

Jáchym, P., Combes, F., Cortese, L., et al. 2014, ApJ, 792, 11

Jáchym, P., Kenney, J. D. P., Sun, M., et al. 2019, ApJ, 883, 145

Leitherer, C., Schaerer, D., Goldader, J. D., et al. 1999, ApJS, 123, 3

Leroy, A. K., Walter, F., Brinks, E., et al. 2008, AJ, 136, 2782

McDonald, M., Veilleux, S., Rupke, D. S. N., et al. 2010, ApJ, 721, 1262

Moore, B., Katz, N., Lake, G., et al. 1996, Nature, 379, 613

Moretti, A., Paladino, R., Poggianti, B. M., et al. 2018, MNRAS, 480, 2508

Muñoz-Mateos, J. C., Boissier, S., Gil de Paz, A., et al. 2011, ApJ, 731, 10

Nehlig, F., Vollmer, B., \& Braine, J. 2016, A\&A, 587, A108

Osterbrock, D. E., \& Ferland, G. J. 2006, Astrophysics of Gaseous Nebulae and Active Galactic Nuclei (CA: University Science Books)

Pappalardo, C., Lançon, A., Vollmer, B., et al. 2010, A\&A, 514, A33

Poggianti, B. M., Moretti, A., Gullieuszik, M., et al. 2017, ApJ, 844, 48

Poggianti, B. M., Gullieuszik, M., Tonnesen, S., et al. 2019, MNRAS, 482, 4466

Roediger, E., \& Hensler, G. 2005, A\&A, 433, 875

Ruszkowski, M., Brüggen, M., Lee, D., et al. 2014, ApJ, 784, 75

Tonnesen, S., \& Stone, J. 2014, ApJ, 795, 148

Vollmer, B., \& Beckert, T. 2003, A\&A, 404, 21

Vollmer, B., \& Leroy, A. K. 2011, AJ, 141, 24

Vollmer, B., Cayatte, V., Balkowski, C., et al. 2001, ApJ, 561, 708

Vollmer, B., Braine, J., Pappalardo, C., et al. 2008, A\&A, 491, 455

Vollmer, B., Soida, M., Chung, A., et al. 2009, A\&A, 496, 669

Vollmer, B., Soida, M., Braine, J., et al. 2012a, A\&A, 537, A143

Vollmer, B., Braine, J., \& Soida, M. 2012b, A\&A, 547, A39

Vollmer, B., Soida, M., Beck, R., et al. 2013, A\&A, 553, A116

Vollmer, B., Pappalardo, C., Soida, M., et al. 2018, A\&A, 620, A108

Vollmer, B., Soida, M., Beck, R., et al. 2020, A\&A, 633, A144

Wiegel, W. 1994, Diploma Thesis, Univ. Heidelberg, Germany

Wiener, J., Pfrommer, C., \& Oh, S. P. 2017, MNRAS, 467, 906

Yagi, M., Yoshida, M., Komiyama, Y., et al. 2010, AJ, 140, 1814

Yagi, M., Yoshida, M., Gavazzi, G., et al. 2017, ApJ, 839, 65 


\section{Appendix A: Additional figures}
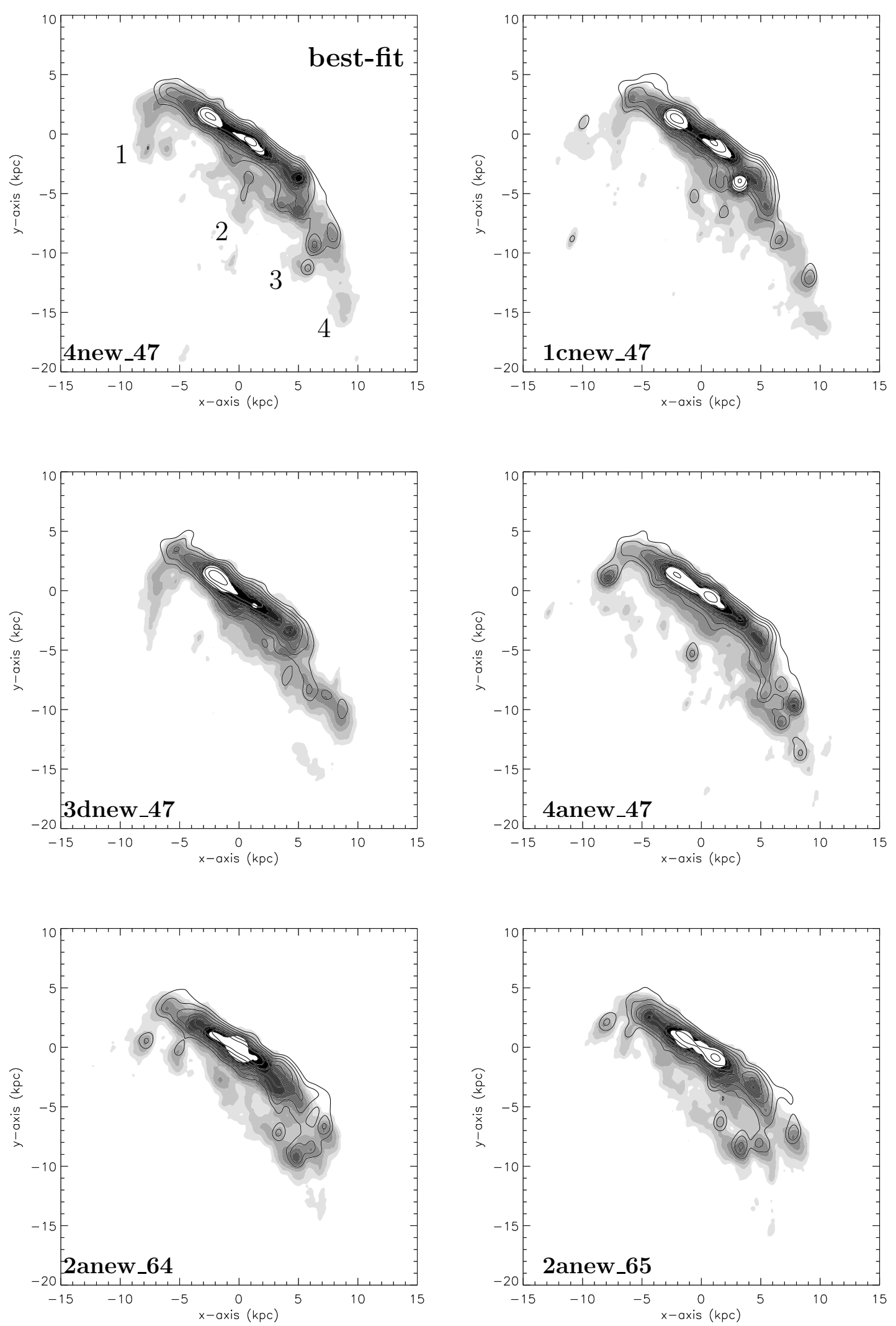

Fig. A.1. H $\alpha$ (grayscale) and NUV (contours) images. Models with a diffuse ISM component are shown. For the spectral fitting, a variable nuisance parameter $\mathrm{Ly}_{\text {scale }}$ was used. 
B. Vollmer et al.: A Virgo Environmental Survey Tracing Ionised Gas Emission (VESTIGE). VIII.
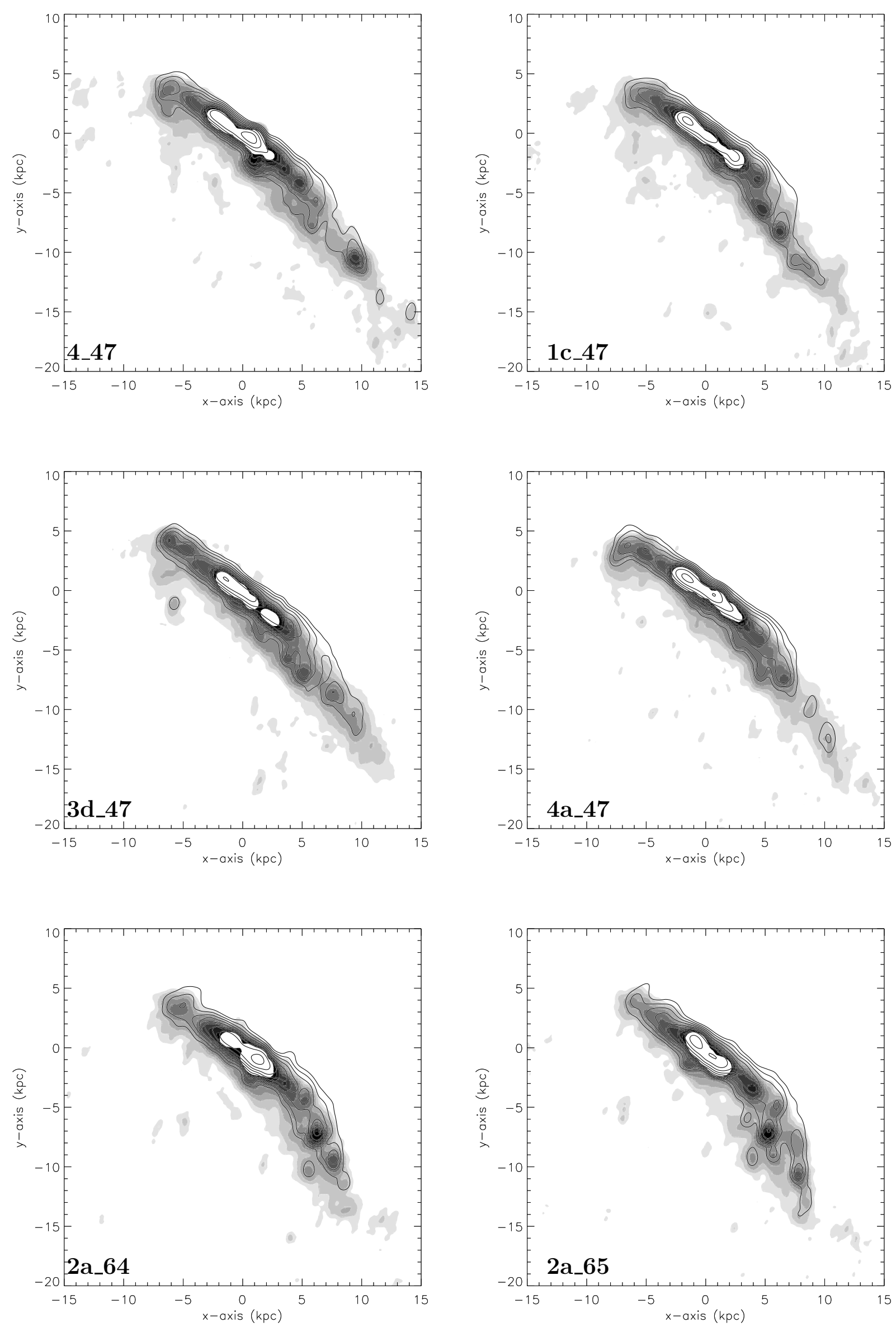

Fig. A.2. Same as Fig. A.1 but without a diffuse ISM component. 
A\&A 645, A121 (2021)
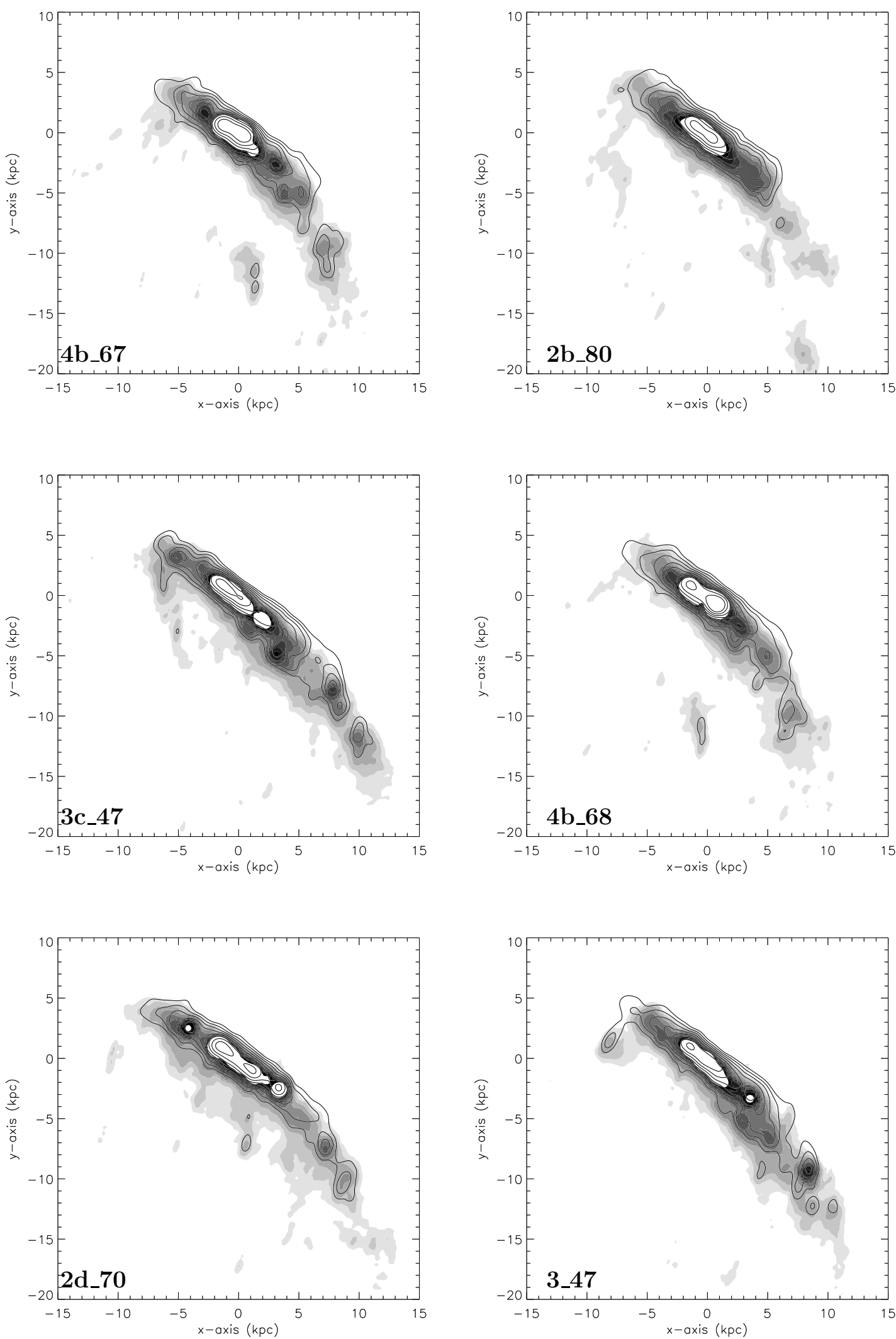

Fig. A.3. H $\alpha$ (grayscale) and NUV (contours) images. Models without a diffuse ISM component are shown. For the spectral fitting, a variable nuisance parameter $\mathrm{Ly}_{\text {scale }}$ was used. 
B. Vollmer et al.: A Virgo Environmental Survey Tracing Ionised Gas Emission (VESTIGE). VIII.

Region 1
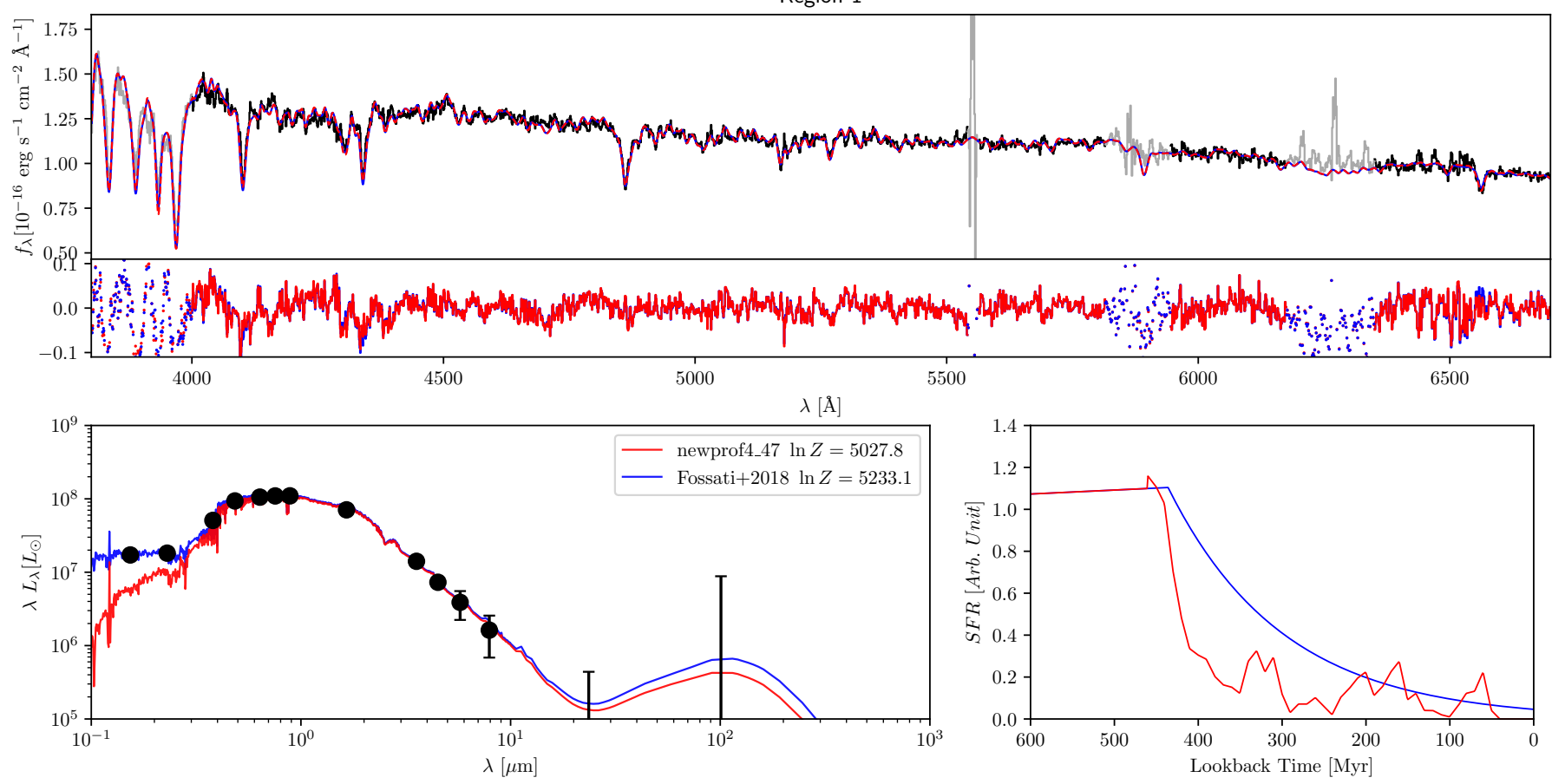

Fig. A.4. Region 1. Upper panel: FORS2 spectrum (black), Fossati et al. model (blue), model 4new_47 with a diffuse ISM component and a variable Ly scale scale parameter (red). Middle panel: fit residuals. Lower-left panel: observed SED (black), Fossati et al. model (blue), model 4new_47 (red). Lower-right panel: SFH, Fossati et al. model (blue), model 4new_47 (red).
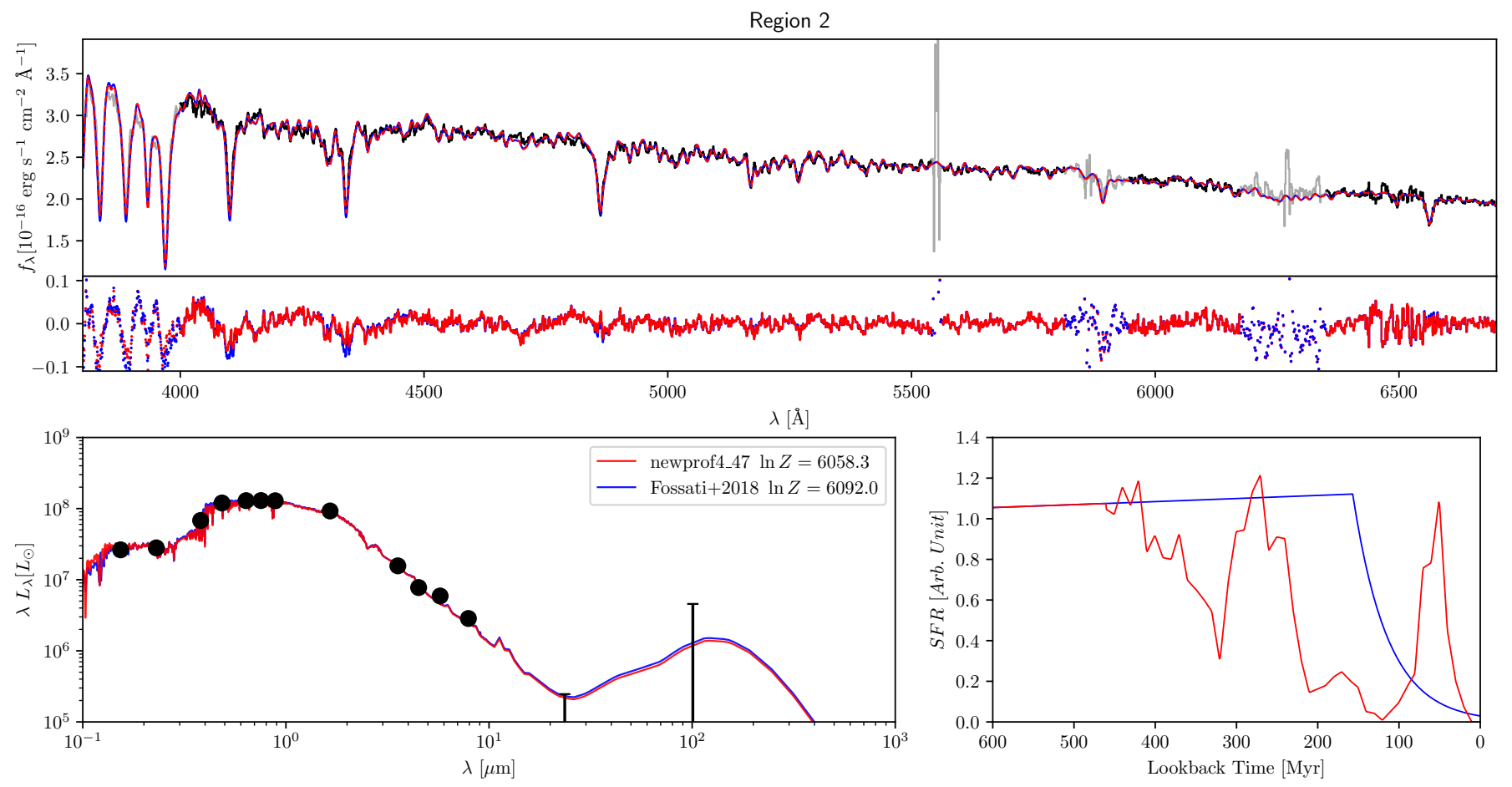

Fig. A.5. Same as Fig. A.4 but for region 2. 
Region 3
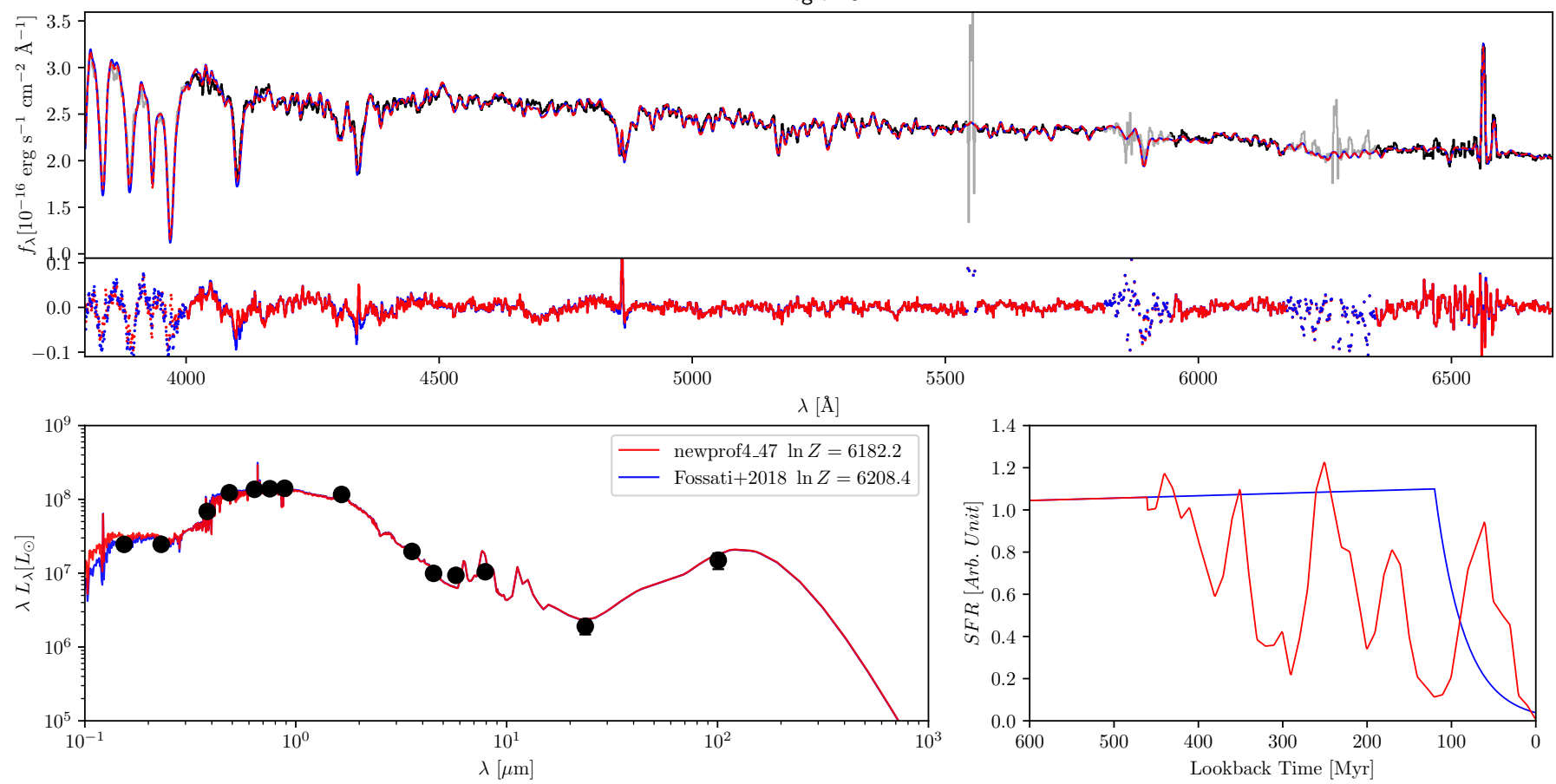

Fig. A.6. Same as Fig. A.4 but for region 3 .

Region 11
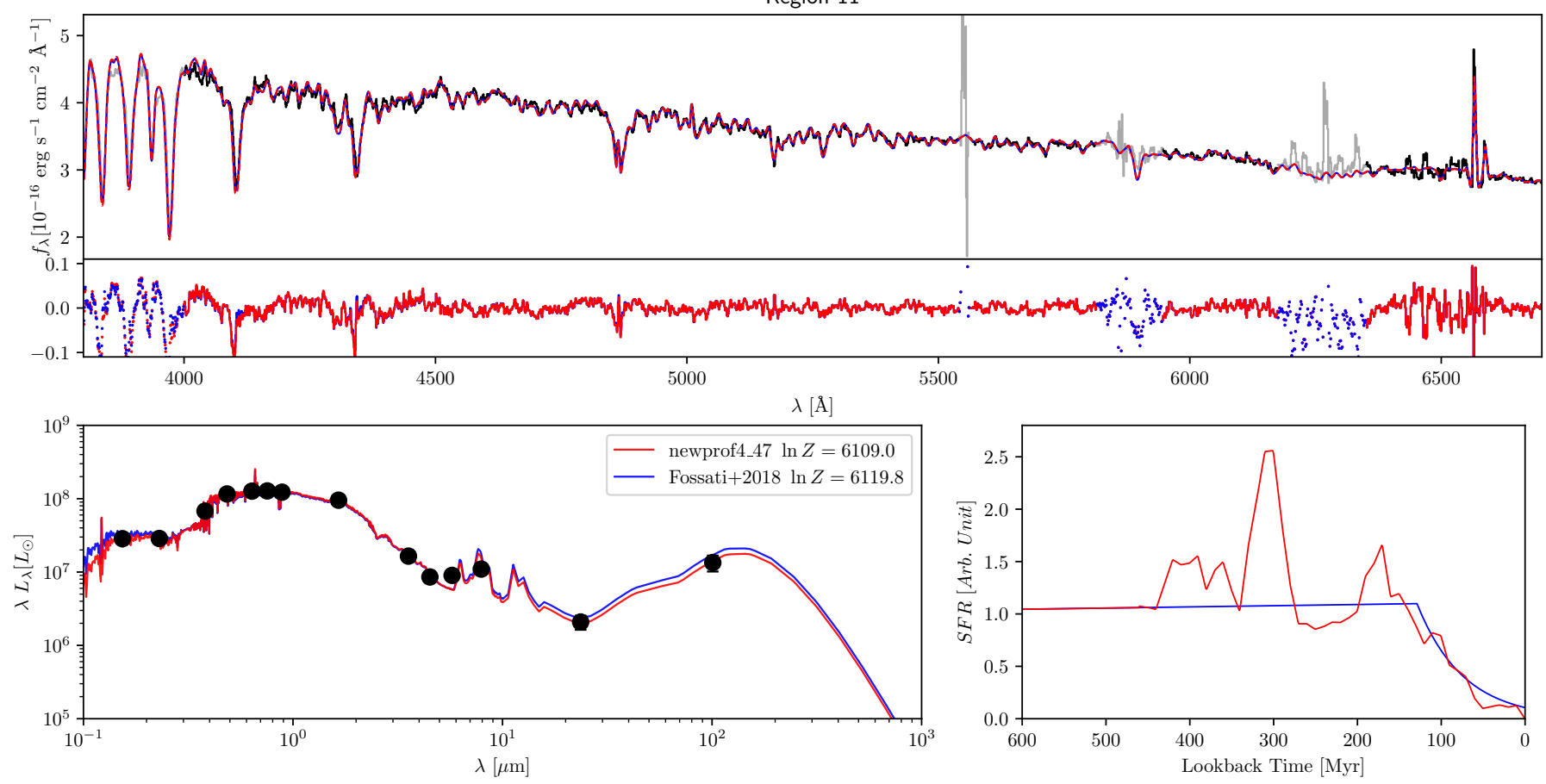

Fig. A.7. Same as Fig. A.4 but for region 11. 
B. Vollmer et al.: A Virgo Environmental Survey Tracing Ionised Gas Emission (VESTIGE). VIII.

Region 12
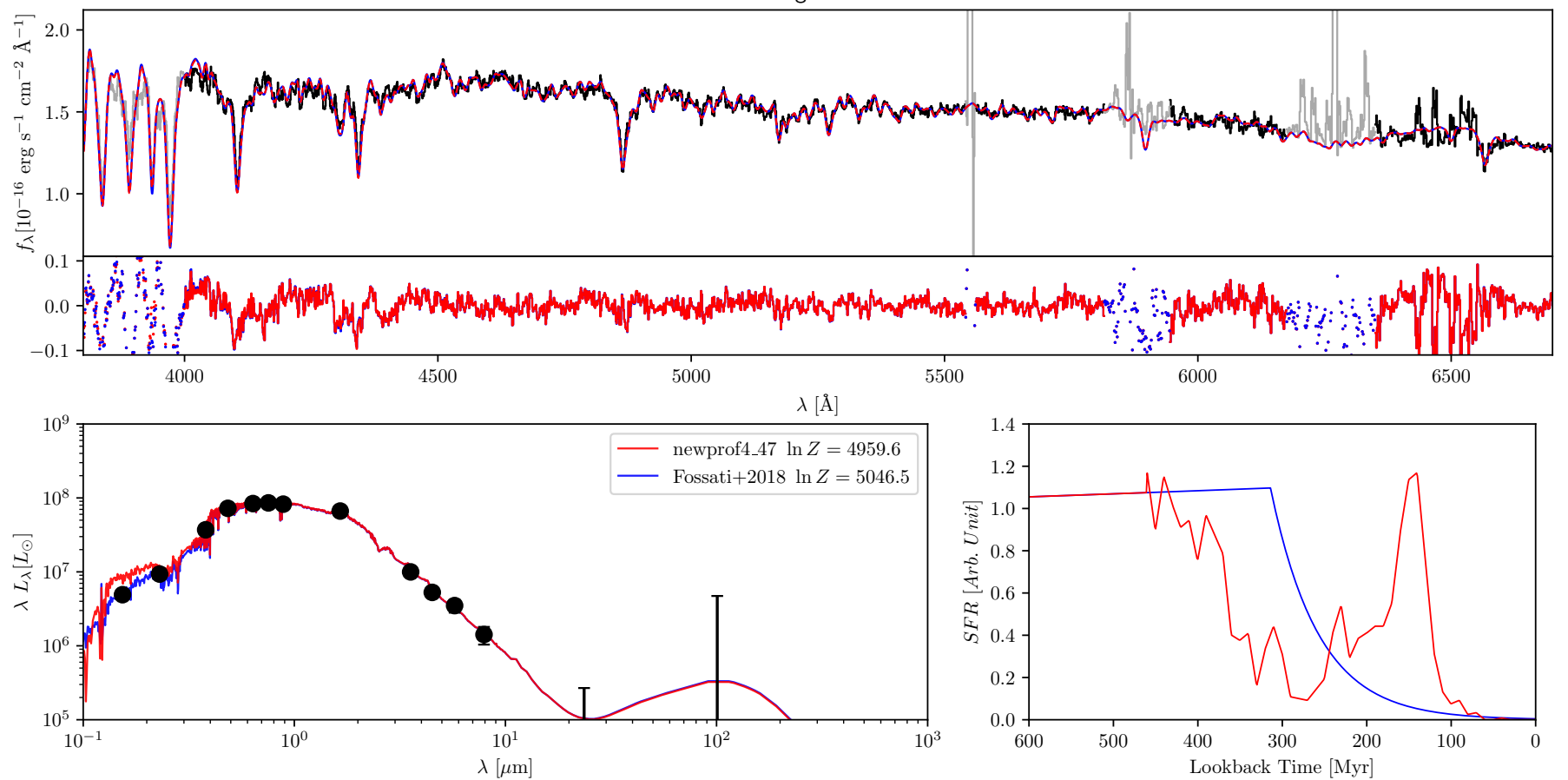

Fig. A.8. Same as Fig. A.4 but for region 12.
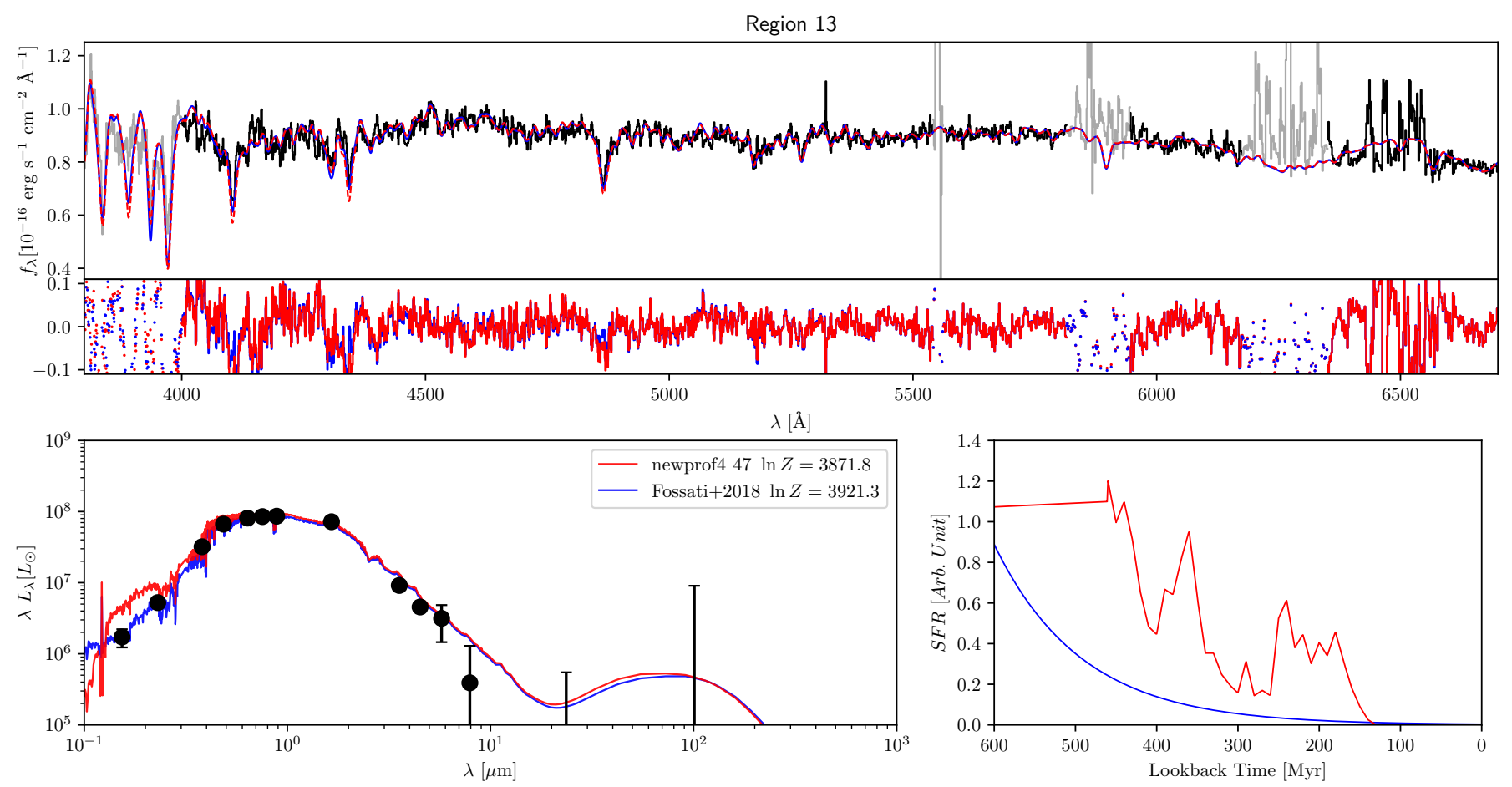

Fig. A.9. Same as Fig. A.4 but for region 13. 


\section{Appendix B: ICM-ISM penetration length}

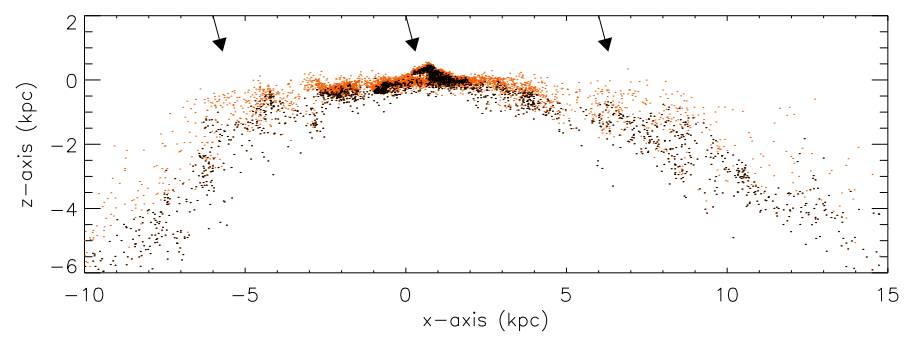

Fig. B.1. Edge-on projection of a slice $(-2 \mathrm{pc}<y<2 \mathrm{pc})$ of model 1 at a time to peak ram pressure of $\Delta t_{\text {peak }}=-100 \mathrm{Myr}$. The black dots represent gas clouds that are protected from the ram pressure wind by other clouds. The gray dots represent gas clouds that are not protected from the ram pressure wind by other clouds. The arrows indicate the wind direction that is opposite to the direction of the galaxy's motion within the ICM.

To estimate the model ICM penetration length into the ISM, we considered a slice $(-2 \mathrm{pc}<y<2 \mathrm{pc})$ of model 1 at a time to peak ram pressure of $\Delta t_{\text {peak }}=-100 \mathrm{Myr}$, when the gas tail is in place. In the chosen projection, the wind direction has no component perpendicular to the image plane. It is therefore possible to estimate the penetration length, which, by definition, depends on the density of the gas clouds. From Fig. B.1, we estimate the penetration lengths to be about $100 \mathrm{pc}, 200-300 \mathrm{pc}$, and about $1 \mathrm{kpc}$ for regions of highest, medium, and lowest densities, respectively. The penetration length, which is the mean free path between clouds, depends on the number of particles and the mass-size relation of the gas clouds. We used the relation found by Vollmer et al. (2001), which leads to realistic gas stripping radii.

\section{Appendix C: Total goodness}

The distributions of the total goodnesses of the simulations with a variable and a constant $\mathrm{Ly}_{\text {scale }}$ parameter and with and without diffuse gas stripping are presented in Fig. C.1. All distributions show a tail to high goodnesses, which comprise $\sim 20$ models.

To give an impression of the quality of the fits with respect to the total goodness $g$, Fig. C. 2 shows two models with $\Delta g=178$. The model with the higher goodnesses reproduces the observed UV and, as a consequence, FIR fluxes significantly better.
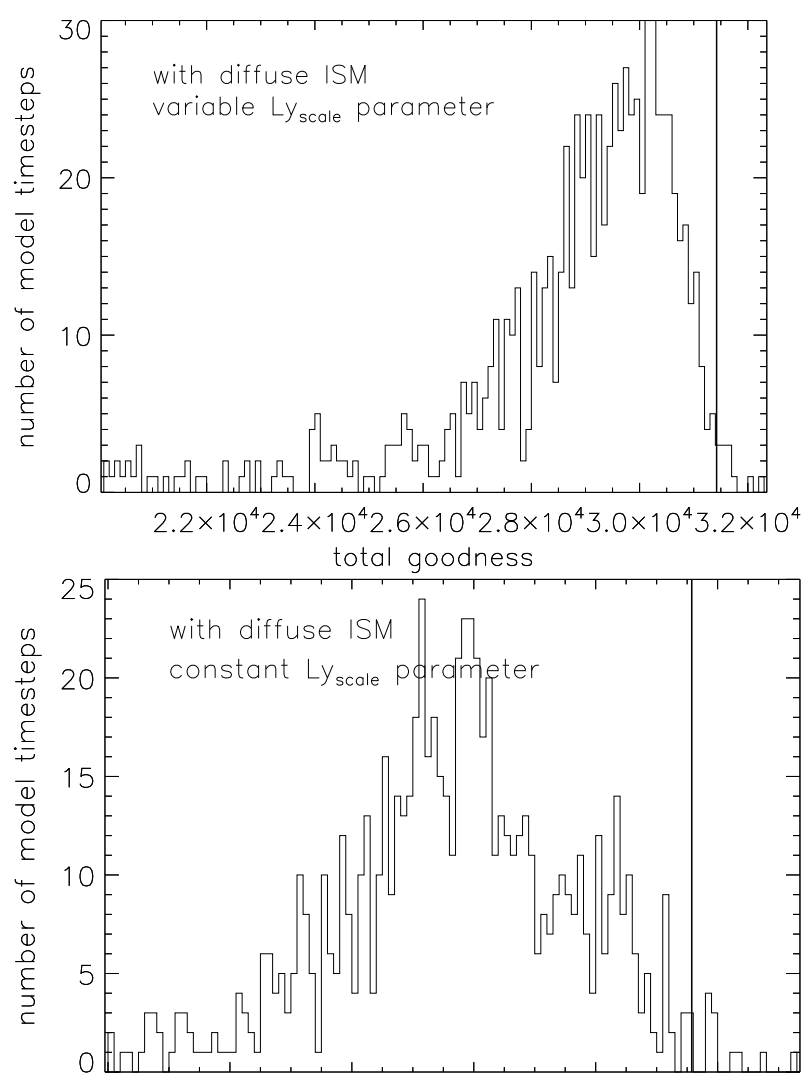

$2.0 \times 10^{4} 2.2 \times 10^{4} 2.4 \times 10^{4} 2.6 \times 10^{4} 2.8 \times 10^{4} 3.0 \times 10^{4}$ total goodness
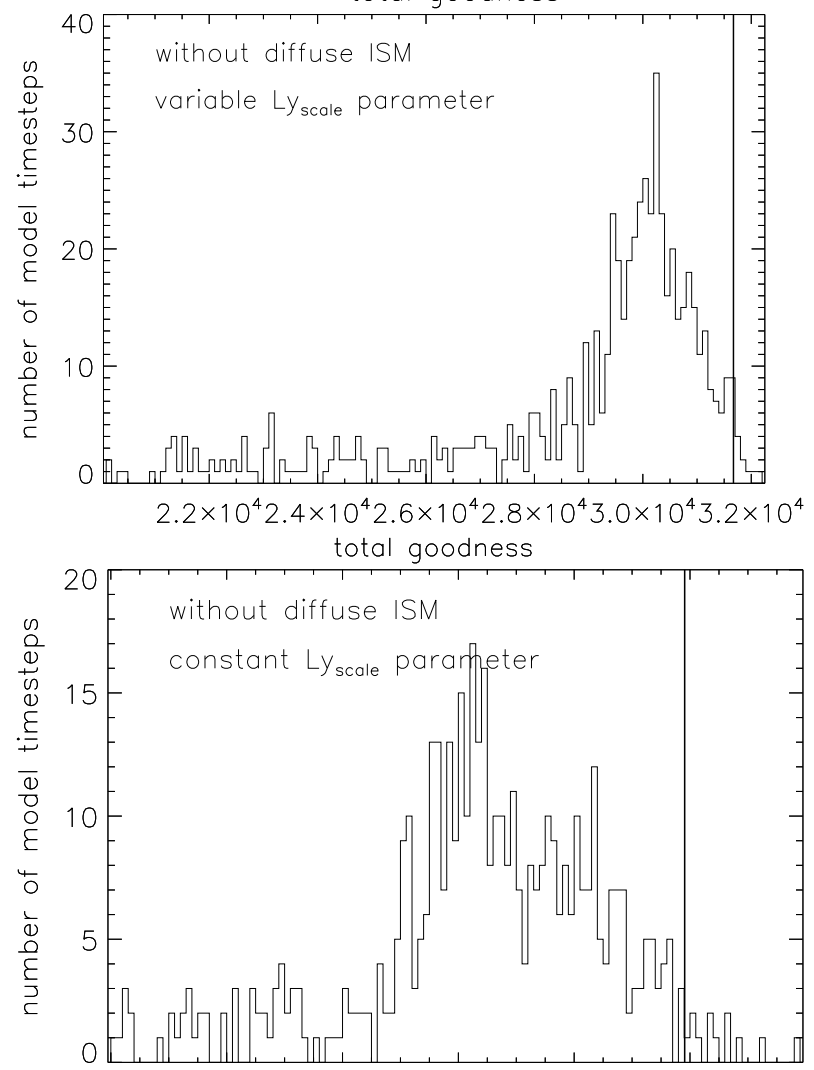

$2.0 \times 10^{4} 2.2 \times 10^{4} 2.4 \times 10^{4} 2.6 \times 10^{4} 2.8 \times 10^{4} 3.0 \times 10^{4}$ total goodness

Fig. C.1. Goodness distributions of the different simulations. The vertical lines delimit the 12 models with the highest goodnesses (see Table 3). 
Region 3
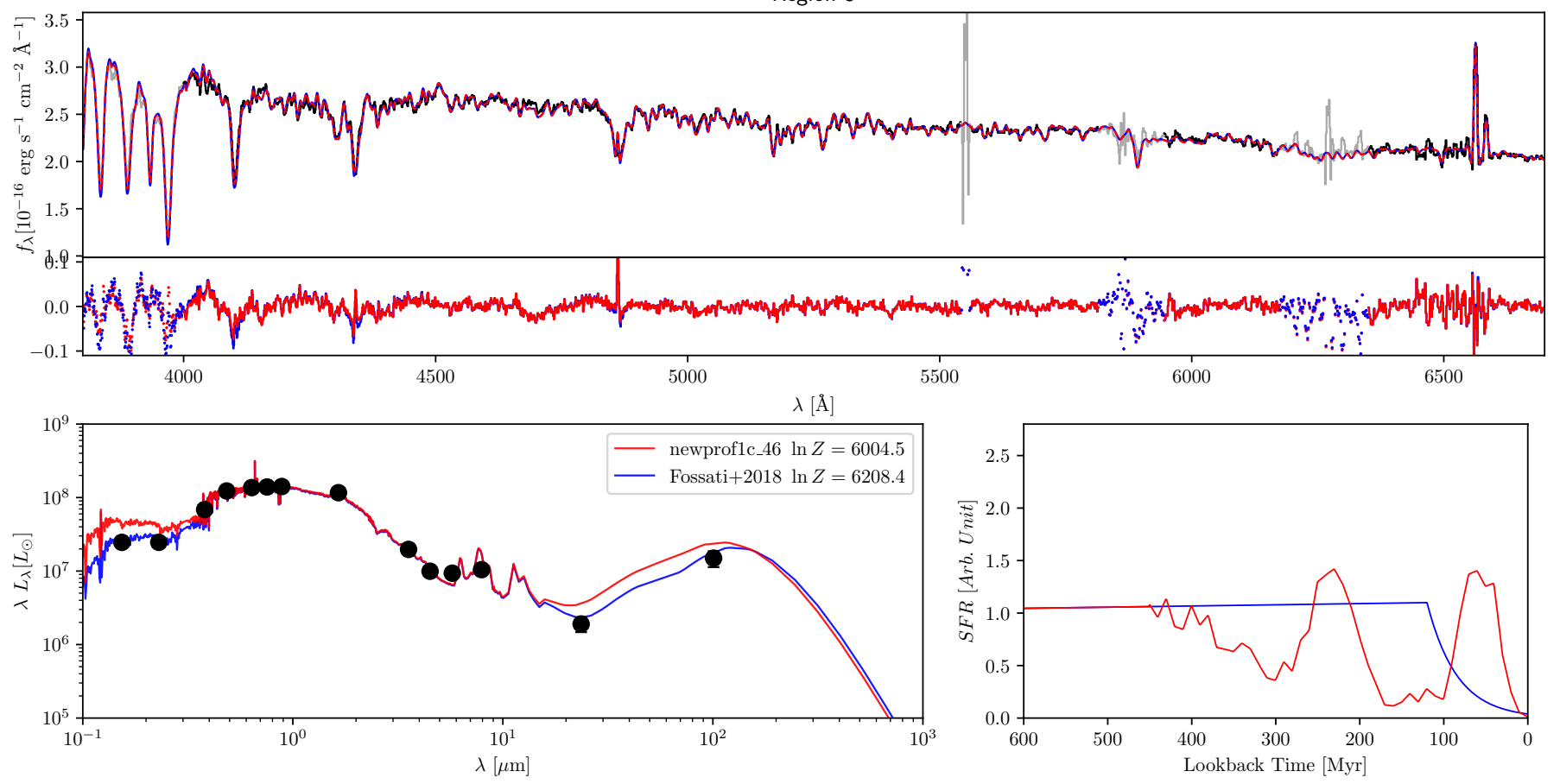

Fig. C.2. Model 1cnew_46, region 3. The total goodness is $g=6004, \Delta g=178$ less than the total goodness of model 4new_47 (Fig. A.6). Upper panel: FORS2 spectrum (black), Fossati et al. model (blue), and model 1cnew_46 (red). Middle panel: fit residuals. Lower-left panel: observed SED (black), Fossati et al. model (blue), and model 1cnew_46 (red). Lower-right panel: SFH, Fossati et al. model (blue), and model 1cnew_46 (red). 
Appendix D: Model fitting with a constant Ly $y_{\text {scale }}$ scale parameter

The model results obtained with a constant nuisance parameter $\mathrm{Ly}_{\text {scale }}$ are presented for the HI and NUV emission dis-
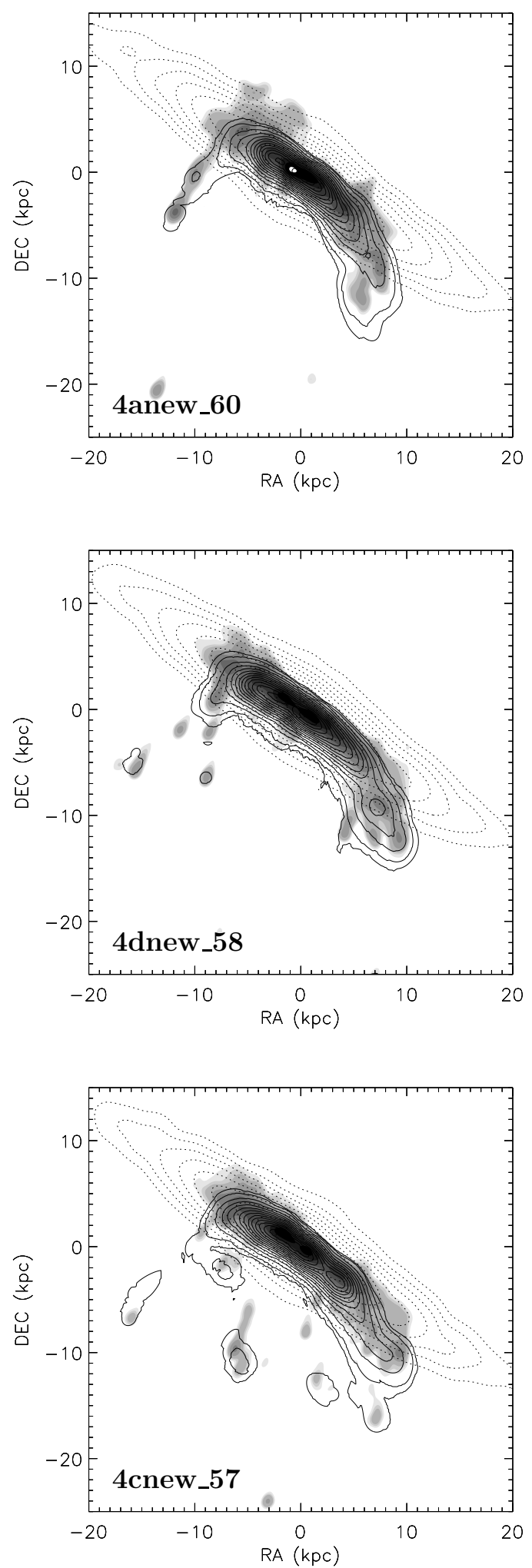

tributions and the $\mathrm{H} \alpha$ emission distribution. For the models with the highest total goodnesses (Table 3), the corresponding observed emission distributions are less well reproduced by these models compared to the models with a variable nuisance parameter.
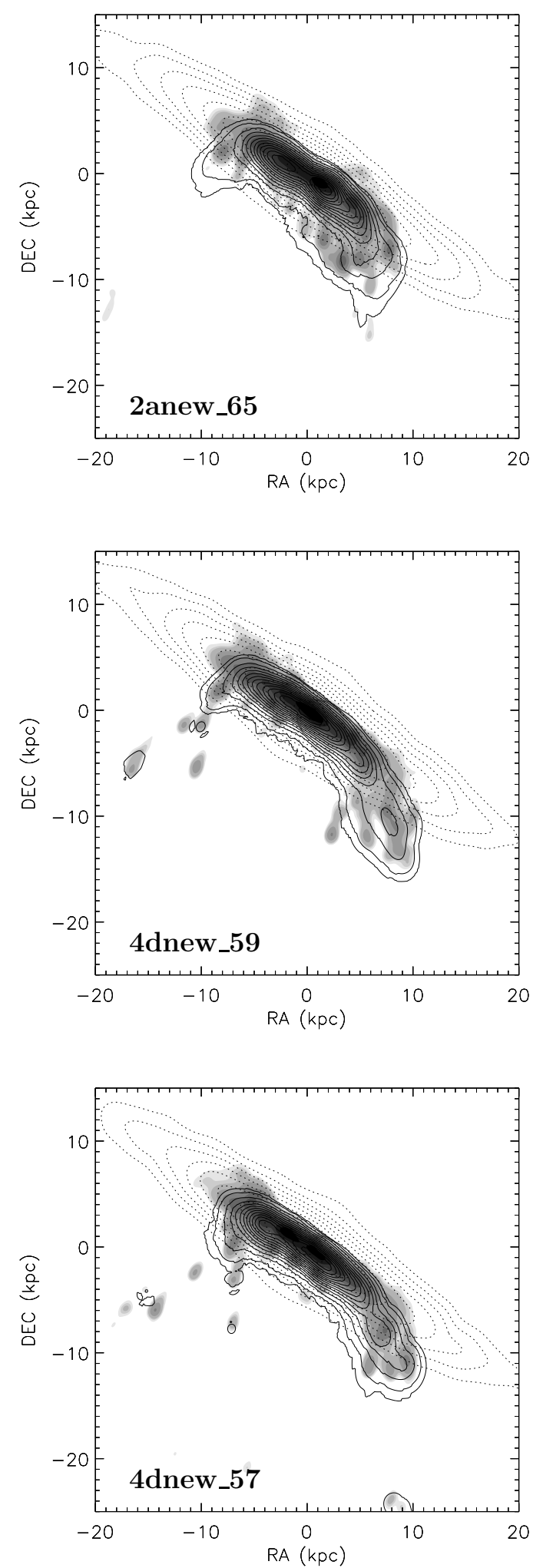

Fig. D.1. Models with a diffuse ISM component. The grayscale represents NUV images, the solid contours represent HI images, and the dotted contours represent stellar images. For the spectral fitting, a constant nuisance parameter $\mathrm{Ly}_{\text {scale }}$ was used. 
B. Vollmer et al.: A Virgo Environmental Survey Tracing Ionised Gas Emission (VESTIGE). VIII.
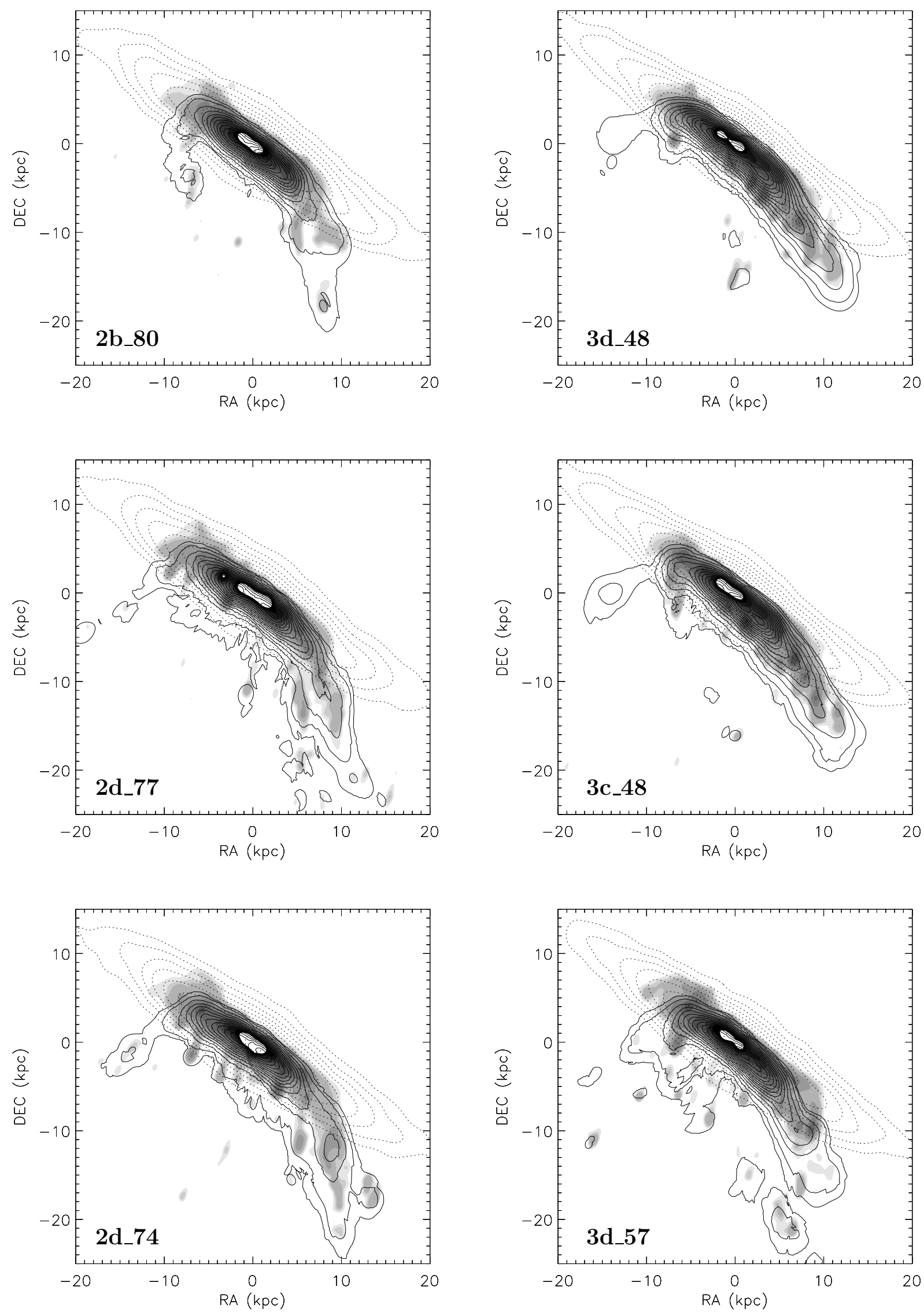

Fig. D.2. Models without a diffuse ISM component. The grayscale represents NUV images, the solid contours represent HI images, and the dotted contours represent stellar images. For the spectral fitting, a constant nuisance parameter $\mathrm{Ly}_{\text {scale }}$ was used. 
A\&A 645, A121 (2021)
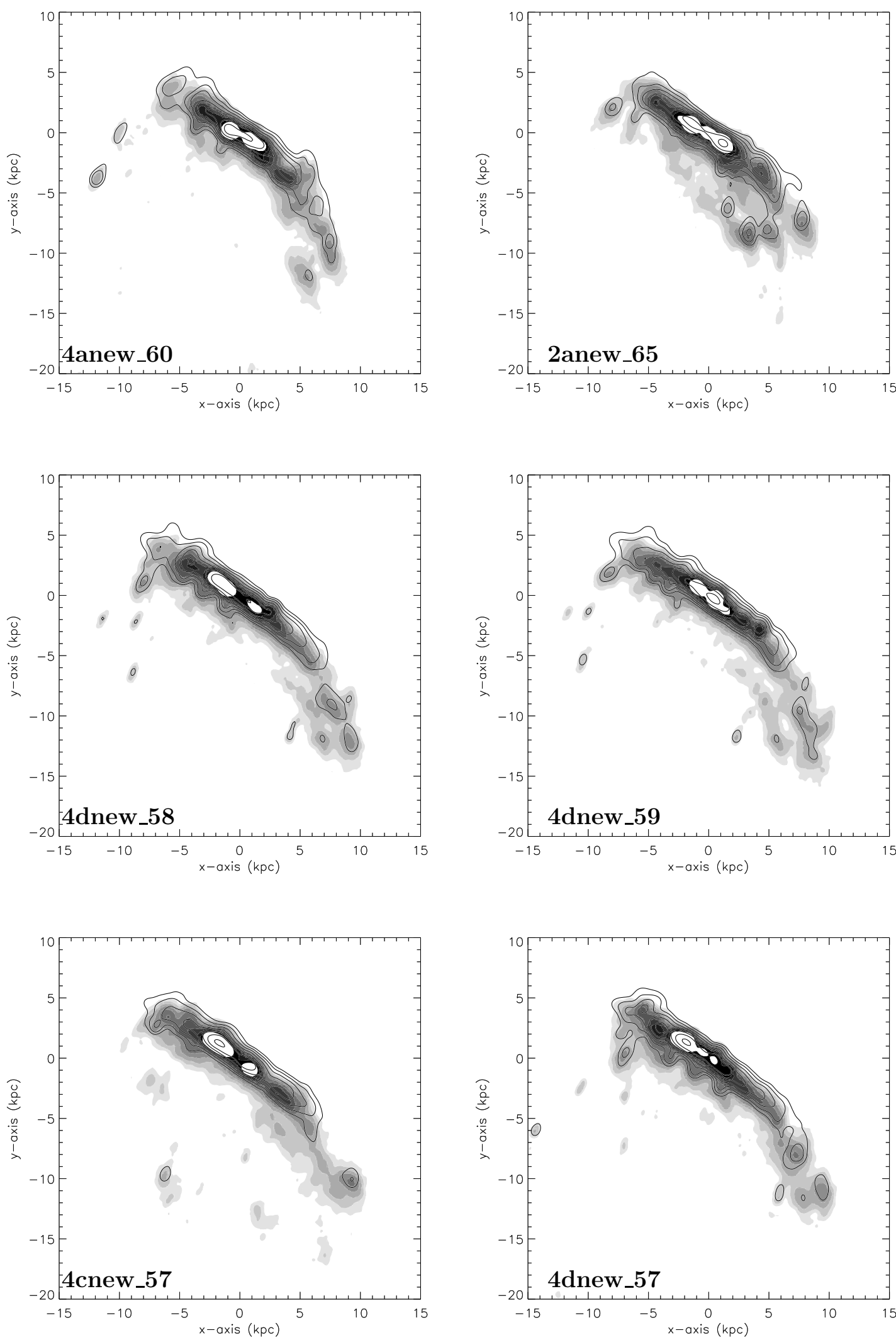

Fig. D.3. Models with a diffuse ISM component. The grayscale represents $\mathrm{H} \alpha$ images and the contours represent NUV images. For the spectral fitting, a constant nuisance parameter $\mathrm{Ly}_{\text {scale }}$ was used. 
B. Vollmer et al.: A Virgo Environmental Survey Tracing Ionised Gas Emission (VESTIGE). VIII.
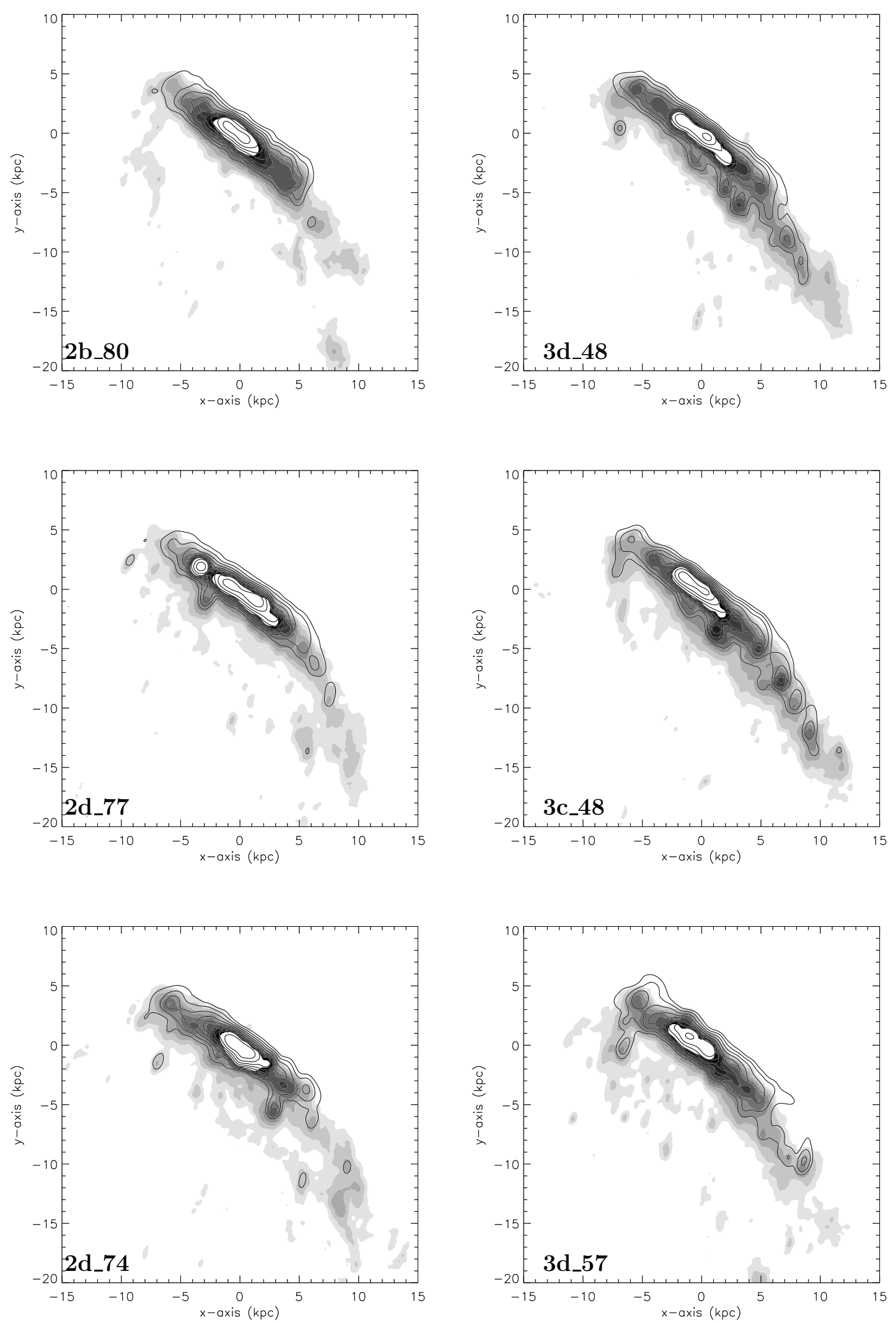

Fig. D.4. Models without a diffuse ISM component. The grascale represents the $\mathrm{H} \alpha$ images and the contours represent the NUV images. For the spectral fitting, a constant nuisance parameter $\mathrm{Ly}_{\text {scale }}$ was used. 